\title{
A minimal model of the Atlantic Multidecadal Variability: its genesis and predictability
}

\section{Hsien-Wang Ou}

\section{Climate Dynamics}

Observational, Theoretical and

Computational Research on the Climate System

ISSN 0930-7575

Volume 38

Combined 3-4

Clim Dyn (2012) 38:775-794

DOI 10.1007/s00382-011-1007-3

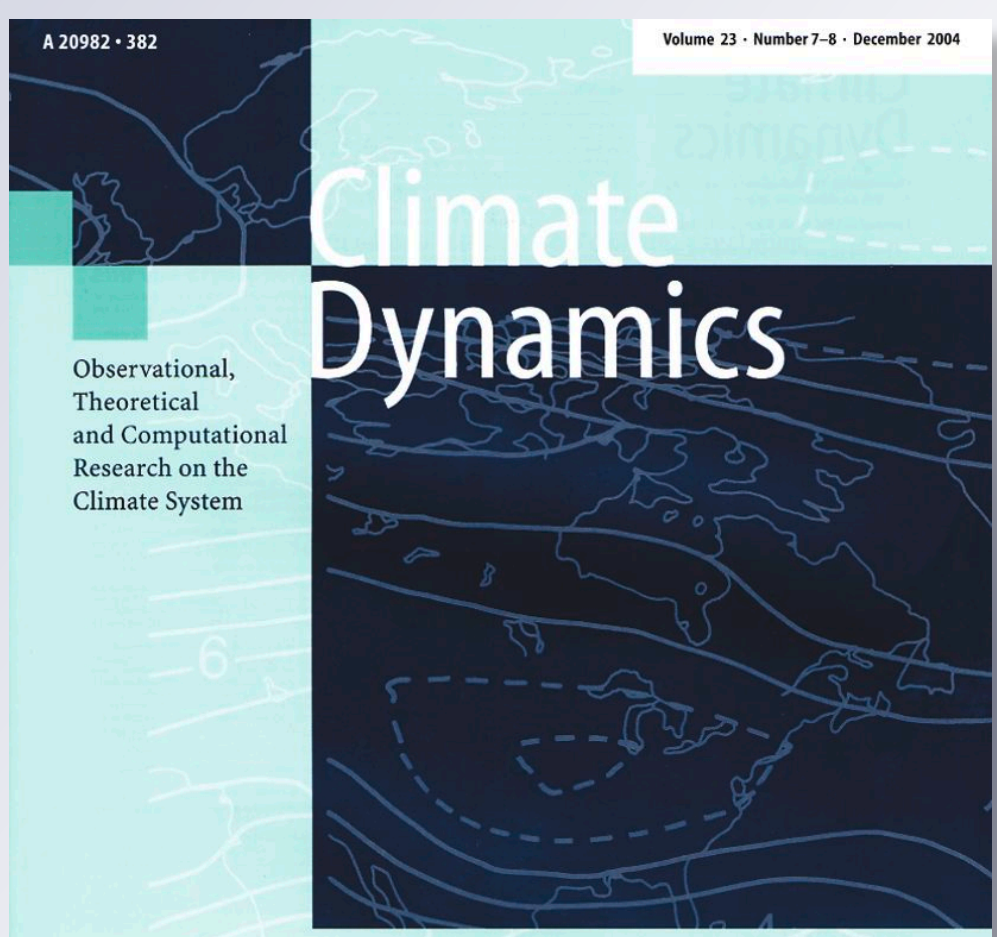

包 Springer 
Your article is protected by copyright and all rights are held exclusively by SpringerVerlag. This e-offprint is for personal use only and shall not be self-archived in electronic repositories. If you wish to self-archive your work, please use the accepted author's version for posting to your own website or your institution's repository. You may further deposit the accepted author's version on a funder's repository at a funder's request, provided it is not made publicly available until 12 months after publication. 


\title{
A minimal model of the Atlantic Multidecadal Variability: its genesis and predictability
}

\author{
Hsien-Wang Ou
}

Received: 16 August 2010/Accepted: 24 January 2011/Published online: 8 February 2011

(c) Springer-Verlag 2011

\begin{abstract}
Through a box model of the subpolar North Atlantic, we examine the genesis and predictability of the Atlantic Multidecadal Variability (AMV), posited as a linear perturbation sustained by the stochastic atmosphere. Postulating a density-dependent thermohaline circulation (THC), the latter would strongly differentiate the thermal and saline damping, and facilitate a negative feedback between the two fields. This negative feedback preferentially suppresses the low-frequency thermal variance to render a broad multidecadal peak bounded by the thermal and saline damping time. We offer this "differential variance suppression" as an alternative paradigm of the AMV in place of the "damped oscillation"- the latter generally not allowed by the deterministic dynamics and in any event bears no relation to the thermal peak. With the validated dynamics, we then assess the AMV predictability based on the relative entropy-a difference of the forecast and climatological probability distributions, which decays through both error growth and dynamical damping. Since the stochastic forcing is mainly in the surface heat flux, the thermal noise grows rapidly and together with its climatological variance limited by the THCaided thermal damping, they strongly curtail the thermal predictability. The latter may be prolonged if the initial thermal and saline anomalies are of the same sign, but even rare events of less than $1 \%$ chance of occurrence yield a predictable time that is well short of a decade; we contend therefore that the AMV is in effect unpredictable.
\end{abstract}

Lamont-Doherty Earth Observatory Contribution Number 7442.

H.-W. Ou ( $\square)$

Department of Earth and Environmental Sciences,

Lamont-Doherty Earth Observatory of Columbia University,

61Route 9W, Palisades, NY 10964, USA

e-mail: dou@1deo.columbia.edu
Keywords Atlantic multidecadal variability - Climate predictability · Relative entropy · Thermohaline circulation . Damped oscillation

\section{Introduction}

Mid-twentieth century warmth in the North Atlantic is a major climatic event of the instrumental period, with the sea surface temperature (SST) in the subpolar region reaching 0.5 K above normal (Deser and Blackmon 1993; Kushnir 1994). Its effects are arguably felt world-wide, including anomalous hydroclimate over the North America and Sahel (Folland et al. 1986; Enfield et al. 2001) and more than 5\% loss of the perennial sea ice in the Arctic (Polyakov et al. 2003). Extending the instrumental records by the proxy data, it is seen that the above warmth is part of the natural cycle that waxes and wanes on multidecadal timescale, as also discernable from their frequency spectrum (Delworth and Mann 2000; Gray 2004). This natural variability was coined the Atlantic Multidecadal Oscillation (AMO) by Kerr (2000) although we shall use the generic "Atlantic Multidecadal Variability" (AMV) without implicating its being an oscillatory mode. Because of its considerable strength and a timescale commensurate with that projected for the anthropogenic signal, the AMV may partially mask the latter (Latif et al. 2004; Knight et al. 2005; Smith et al. 2007; Ting et al. 2009); understanding the genesis and predictability of the AMV thus is important in differentiating and assessing the human-induced climate change.

In contrast to the decadal Arctic Oscillation (AO), the AMV does not exhibit similar causal relation with the atmospheric variables, leading to the suggesting that its genesis is oceanic in origin (Bjerknes 1964; Deser and 
Blackmon 1993; Kushnir 1994). Two probable but distinct processes have been postulated: one pertains to the selfpropelling thermal anomaly when coupled to the regional overturning circulation (Te Raa and Dijkstra 2002), which is inherently three-dimensional with its timescale related to the basin-crossing time. While the feature has been demonstrated in isohaline ocean models (Greatbatch and Zhang 1995; Saravanan and McWilliams 1997; Huck et al. 1999; Te Raa and Dijkstra 2002), its relevance to the observed AMV is less clear since the latter is indexed by the basinscale SST anomaly (Enfield et al. 2001; Delworth et al. 2007), which might not register the signal from a travelling thermal wave. The other process, which has manifested prominently in coupled general circulation models (GCMs), involves the thermohaline circulation (THC) interacting with the density anomaly particularly in the subpolar region (Delworth and Greatbatch 2000; Latif et al. 2004; Knight et al. 2005; Dong and Sutton 2005). Differing from the above thermal waves, the process may operate on the two-dimensional meridional plane and in which both temperature and salinity variations play key roles. Its timescale varies widely among models (Delworth et al. 2007; Danabasoglu 2008) but has been loosely attributed to the THC overturning time (Winton and Sarachik 1993; Te Raa and Dijkstra 2003; Dong and Sutton 2005; Sevellec et al. 2006).

Since the AMV is associated with the basin-scale SST anomaly and the THC is the oceanic transport mechanism operating on this scale, it is to be expected that the THC should play a central role in the AMV - a prevailing view that is also taken by the present study. But besides the ocean heat, the THC also redistributes the salt, and together they determine the large-scale density contrast, which in turn may regulate the strength of the THC. Incorporating these essential couplings, even simple box models have exhibited highly varied behavior due in essence to a salinity contrast that is counter to the density stratification (Stommel 1961); this configuration is itself a direct consequence of the poleward moisture transport driven by the differential heating (Marotzke and Stone 1995; Ou 2007), which has planted the root of the instability. In the finiteamplitude regime, the system behavior includes the multiequilibria (thermal, saline and saddle modes), Hopf bifurcation, limit cycle and hysteresis-all have been invoked to explain large swings in the paleoclimate (Broecker et al. 1990; Rahmstorf 2002; Clark et al. 2002; Dijkstra 2005).

Comparing to these paleoclimate events, the AMV represents merely a small perturbation of the thermal state that characterizes the Holocene hence likely governed by the linear dynamics, and a widely held view is that it is a damped oscillation sustained by the stochastic atmosphere (Griffies and Tziperman 1995; Delworth and Greatbatch
2000; Dong and Sutton 2005). Although the stochastic forcing is well justified on account of the atmospheric chaos (Lorenz 1969), whether the deterministic dynamics allows oscillatory modes and whether such modes correspond to the observed AMV have not been firmly established. Nor have the key parameters been explicitly identified that control the timescale or the strength of the AMV.

Because of the stochastic nature of the AMV, there arises the question of its predictability. A common approach is through ensemble forecasts using GCMs (Griffies and Bryan 1997a; Collins et al. 2006), which is computationally demanding because of the need of large ensemble of long integrations to garner adequate statistics. Alternatively, the inverse modeling has been applied to the observed or modeled timeseries to extract the deterministic dynamics (Tziperman et al. 2008), which, however, cannot be easily linked to the physical parameters that govern the observed system, and being diagnosed from a single (presumably stationary) realization, it is difficult to generalize the finding to a changed environment. For a more general assessment of the predictability, a preferred approach thus is to derive the dynamical operator based on physical balances, and box models of varying complexity have been used for this purpose (Griffies and Tziperman 1995; Lohmann and Schneider 1999; Tziperman and Ioannou 2002). Since the model physics is highly truncated, it needs to be first validated by the observed variability before inferences on the predictability can be drawn, a step, however, not sufficiently bridged; and then the more pertinent predictability measures from the information theory, such as the relative entropy, have not been widely employed in the analyses.

Motivated by above shortfalls in addressing the genesis and predictability of the AMV, we consider a simple box model to elucidate the minimal physics and to clarify some fundamental issues. When applied to the parameter regime of the actual system, this minimal model nonetheless provides a reasonable account of the observed phenomenon and in the process raises significant questions on its prevailing interpretation as a damped oscillation. And with the validated dynamics, we then revisit the predictability problem using the relative entropy, which shed additional light on the AMV predictability and underscores its severe limitations.

For the organization of the paper, we first formulate in Sect. 2 a box model of the North Atlantic, culminating in a pair of coupled equations governing the thermohaline perturbations of the subpolar convective region. In Sect. 3, we examine their spectral properties, including a multidecadal thermal peak, which compares favorably with the observed AMV, and we propose a robust mechanism for its genesis. In Sect. 4, we consider the predictability of the AMV based on the relative entropy, and discuss its 
optimization and parameter dependence. In Sect. 5, we summarize the main findings and provide additional discussion.

\section{Box model}

\subsection{Formulation}

We consider a box model of the North Atlantic, as sketched in Fig. 1. The two boxes (designated 1 and 2) correspond to the warm and cold watermasses, which are separated by the main thermocline and its surface outcrop (the subtropical front), arguably a minimal description of the observed ocean. To assess the finite-amplitude climate behavior, such as multi-equilibria, one obviously needs to treat the thermohaline properties of both boxes as prognostic, but to examine small perturbation of a given base state, such as the AMV, additional simplifications are possible. In particular, the observed AMV is characteristically monopolar with its maximum signal concentrated in the upper depths of the subpolar convective region (Deser and Blackmon 1993; Kushnir 1994), which define our cold box. This anomaly pattern also emerges in model simulations (e.g. Delworth et al. 1993), which can be attributed to the small volume of the cold box compared with the warm watermass that spans two hemispheres and its proximity to the Arctic Ocean where the primary freshwater perturbation is originated (see later discussion). As a minimal model, we shall thus treat the warm box as a vast unvarying reservoir that anchors the thermohaline perturbations of the cold box. One of course may include the warm-box properties as unknowns-as in most box models, which would complicate the mathematics but does not alter the basic physics; and moreover we shall demonstrate that our

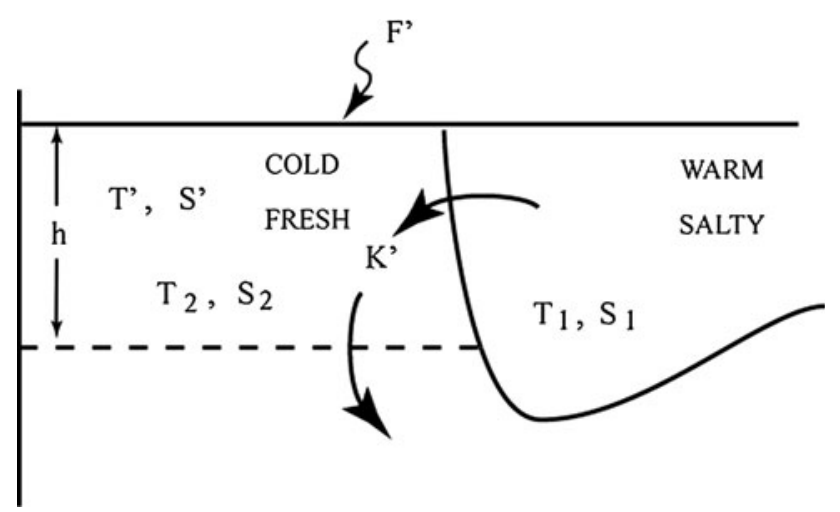

Fig. 1 The box model of the AMV. The cold box represents the upper part (depth $h$ ) of the sinking region where the thermohaline perturbations (primes) are induced by the stochastic surface heat flux $\left(F^{\prime}\right)$ and coupled through a density-dependent thermohaline circulation (THC) denoted by $K^{\prime}$ single-box model, even with fewer tunable parameters, is capable of capturing the essential AMV variability.

After removing global-mean surface fluxes, the heat balance of the cold box is governed by

$h \frac{d T_{2}}{d t}=F_{r}-C\left(1-\mu^{*}\right) T_{2}+K\left(T_{1}-T_{2}\right)+F^{\prime}$,

where $h$ is the depth of the cold box (see Fig. 1), $F_{r}$, the radiative flux, $C$, the air-sea transfer coefficient defined by

$C \equiv \frac{C_{d}\left|u^{\prime}\right| \rho_{a} C_{p, a}}{\rho_{o} C_{p, o}}$

with $C_{d}$ being the drag coefficient, $\left|u^{\prime}\right|$, the turbulent wind speed, $\rho_{a}$ and $C_{p, a}$ the density and specific heat capacity of the surface air, and $\rho_{o}$ and $C_{p, o}$, their counterparts for the water; $\mu^{*}$ measures the coupling strength of the surface-air to the ocean temperature, $K$, the THC transport divided by the surface area of the cold box (referred as THC for short), and $F^{\prime}$, the stochastic surface heat flux (both radiative and stochastic fluxes have been divided by $\rho_{o} C_{p, o}$ ). This equation states that the heat content may be altered, sequentially on the right-hand side, by the radiative and convective fluxes at the surface, the THC, and the stochastic surface heating. Although the stochastic forcing stems from atmospheric eddies, it is organized by teleconnection into large-scale pattern (Wallace and Gutzler 1981) to impart a net forcing on the cold box, and based on atmospheric GCM runs, this stochastic forcing is assumed to be white noise (Saravanan and McWilliams 1997; Delworth and Greatbatch 2000).

Since there is no coupling of the hydrological cycle to the salinity, the latter is subjected to a simpler balance

$h \frac{d S_{2}}{d t}=-F_{w} S_{o}+K\left(S_{1}-S_{2}\right)$,

where $F_{w}$ is the freshwater input, which consists of P-E over the cold box and fluxes from the Arctic Ocean (in both solid and liquid forms), and $S_{o}$ is the reference ocean salinity. Invoking model results (Delworth and Greatbatch 2000; Cheng et al. 2004), we have neglected the stochastic freshwater forcing. One reason for this difference from the thermal forcing is that the anomalous surface air temperature (SAT) associated with atmospheric eddies immediately impacts the surface heat flux but the anomalous moisture they carry does not necessarily precipitate since it involves cloud physics of additional degrees of freedom; and this stochastic freshwater forcing in any event is small compared with that associated with the varying THC (Griffies and Tziperman 1995).

The THC, being a proxy for the meridional transport mechanism, can be facilitated by eddy exchange across the subtropical front (Lozier 2010), but for models that do not resolve eddies, it necessarily manifests as a Meridional 
Overturning Cell (MOC) driven by the density contrast. Being an ageostrophic flow, the THC depends strongly on the diapycnal diffusivity (Bryan 1987); the latter thus is in effect tuned to yield the climatological thermal field (Wunsch and Ferrari 1994) and-with that-the required THC. With the diapycnal diffusivity fixed as such, the THC then varies with the density contrast, as indeed seen in numerical models (Delworth et al. 1993). Notwithstanding the empirical basis of this linkage (see further discussion in Sect. 5), we postulate nonetheless

$K \propto \Delta \rho$,

$\propto-\alpha \Delta T+\beta \Delta S$

where $\Delta$ indicates the difference between the warm and cold boxes and $\alpha$ and $\beta$ are the thermal expansion and saline contraction coefficients, respectively. Recalling that the warm-box properties are assumed fixed, the perturbation in the THC thus is proportional to the density anomaly of the cold box. One notes incidentally that it is because of the assumed homogeneity of the cold box that there is no lag between the THC and the density anomaly; in a more refined model, there could be a lag if one defines the density anomaly as that averaged over the cold box, but as we shall see later (Sect. 2.1), this lag can be absorbed into the overturning time hence does not constitute a limitation of the model.

Since we are concerned with small perturbations about the climatological means, we decompose the variables accordingly so that

$T_{2}=\bar{T}_{2}+\Delta \bar{T} T^{\prime}$

$S_{2}=\bar{S}_{2}+\Delta \bar{S} S^{\prime}$,

$K=\bar{K}\left(1+K^{\prime}\right)$,

where the overbars and primes denote, respectively, the climatological means and the fractional perturbations. We shall consider the linear regime when the fractional perturbations are small compared with unity, in which case (4) becomes

$K^{\prime}=\frac{-T^{\prime}+\gamma S^{\prime}}{1-\gamma}$,

where $\gamma \equiv(\alpha \Delta \bar{T})^{-1}(\beta \Delta \bar{S})$ is the inverse density-ratio, a measure of the degree to which the salinity contrast reduces the density stratification associated with the thermal contrast. It is seen that, as a chief advantage of using the fractional perturbations, (8) is independent of the proportional constant linking the THC to the density anomaly (4), which thus need not be specified.

Freshwater flux $F_{w}$ in (3) can be perturbed by the SST. Warming of the cold box, for example, would increase the precipitable water in the overlying atmospheric column on account of the Clausius-Clapeyron equation, but it also reduces the differential heating of the atmosphere by the SST hence the poleward atmospheric energy transport, which would then counter the above effect (Ou 2007). The P-E over the cold box may also vary with the zonal convergence of the moisture transport (Timmermann et al. 1998), whose quantitative linkage to the SST, however, remains unknown. In contrast to these uncertain effects on the P-E, it is well demonstrated in climate models that a positive SST anomaly would increase the freshwater flux from the Arctic Ocean (Delworth et al. 1997; Holland et al. 2001; Jungclaus et al. 2005). This can be through the warming-induced ice melt, which would freshen the Arctic Water and increase its discharge to the subpolar oceans through the East Greenland Current (Hakkinen 1993) or it can be through an enhanced atmospheric cyclonicity that drives greater ice export through the Fram Strait (Walsh and Chapman 1990; Dima and Lohmann 2007). To incorporate this coupling, we introduce a freshwater coupling coefficient $v$ and set

$F_{w}=\bar{F}_{w}\left(1+v T^{\prime}\right)$

so that warming of the cold box would augment the freshwater flux it receives. Again, for simplicity, we have neglected the time lag between the two, which is not expected to materially alter the model results.

To complete the non-dimensionalization, we scale the time by the overturning time (hence subscripted "o") of the cold box defined by

$t_{o} \equiv h / \bar{K}$,

and the stochastic surface heat flux by

$F^{\prime}=(\bar{K} \Delta \bar{T}) q^{\prime}$.

Substituting (5)-(11) into (1) and (3), the fractional perturbations are then governed by the coupled equations

$\dot{T}^{\prime}=c_{1} S^{\prime}-\alpha_{1} T^{\prime}+q^{\prime}$,

$\dot{S}^{\prime}=-c_{2} T^{\prime}-\alpha_{2} S^{\prime}$,

where the coefficients have the expressions

$c_{1}=\frac{\gamma}{1-\gamma}$,

$c_{2}=\frac{1}{1-\gamma}+v$,

$\alpha_{1}=1+\frac{1}{1-\gamma}+\mu$

and

$\alpha_{2}=1-\frac{\gamma}{1-\gamma}$,

with

$\mu \equiv C(1-\mu *) / \bar{K}$ 
referred as the thermal damping coefficient. One is reminded that a stronger SAT-coupling to the SST (that is, greater $\mu^{*}$ ) implies a smaller $\mu$ hence weaker thermal damping. For convenience, the $c$ 's and $\alpha^{\text {'s }}$ are referred as thermohaline coupling and damping constants, respectively, and since all these dimensionless parameters, including $\alpha_{2}$ (see Sect. 2.3), are positive, the essential role of the THC is readily discerned, which can be illustrated through feedback loops, as discussed next.

\subsection{Feedback loops}

The unity terms in the damping constants $\alpha^{\text {ss }}$ represent "inherent" damping by a constant THC, so for example a positive anomaly in either temperature or salinity would decrease the respective poleward flux from the warm box to damp that anomaly. An interactive THC, however, generates additional effects, which are succinctly summarized in Fig. 2. The upper arrows indicate the density effect on the THC and the lower arrows, the THC effect on the thermohaline perturbations, with the attached signs referring to the relative signs of the changes linked by the arrows. It is seen for example warm (salty) anomaly would lighten (densify) the cold box to decrease (increase) the THC - hence the negative (positive) signs in the respective upper arrows. An increasing THC on the other hand would yield both warmer and saltier cold box, hence both the positive signs in the lower arrows.

Tracing the closed loops, one sees that the THC coupling to the thermal anomaly forms a "thermal" loop (the left one) of net negative sign, which thus would enhance the thermal damping: that is, a warm anomaly for example would weaken the THC to reduce the heat it carries into the cold box, damping the initial warmth. On the other hand, the THC coupling to the saline anomaly forms a positive "saline" loop (the right one) that would reduce the saline damping, so for example a salty anomaly would strengthen the THC to inject more salt into the cold box, thus reinforcing the initial salty anomaly. Since the thermal

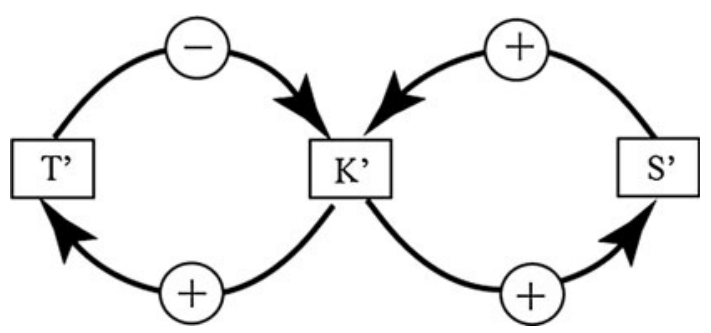

Fig. 2 The feedback loops facilitated by the THC coupling to the thermohaline anomalies. The upper arrows indicate the density effect on the THC and the lower arrows, the THC effect on the thermohaline perturbations. The attached signs refer to the relative signs of the perturbations linked by the arrows damping constant $\alpha_{1}$ is already greater than its saline counterpart $\alpha_{2}$ due to the air-sea flux as entailed in $\mu$ (comparing [16] and [17]), the above opposite effect of the THC further differentiates the thermal and saline damping, resulting in, as we shall see later, an order of magnitude difference in their damping time. The positive saline loop is of course the source of the well-known saline instability and moreover it is seen from (17) that if the inverse density-ratio approaches $1 / 2$ (still well short of the gravitational instability), the saline damping would vanish, leading to saline catastrophe and possible reorganization of the climatic state (Walin 1985). This limit, however, has little relevance to the AMV, which is a small perturbation of the stable thermal state for which the inverse density-ratio is by stipulation less than $1 / 2$ and hence $\alpha_{2}$ is positive, as alluded to earlier.

The thermal and saline loops also combine to form a larger "thermohaline" ( $\infty$-shaped) loop, which has a net negative sign hence represents a negative feedback between the thermal and saline fields, so for example a warm anomaly in the cold box would weaken the THC to freshen the cold box, which would further weaken the THC to counter the initial warmth. This negative feedback is the source of the damped oscillation widely postulated for the AMV, but there is a crucial factor that is not sufficiently recognized: the THC that facilitates the negative feedback is the same THC that would regulate the damping, so it's not obvious that oscillatory eignenmodes actually exist in a realistic oceanic regime; nor is it ascertained that, even if such oscillatory modes exist, they correspond to the observed thermal peak that defines the AMV - both in fact answered in the negative based on later analyses. Incidentally, since all the loops in Fig. 2 are closed by the lower arrows that involve the overturning time, the latter may absorb the neglected lag between the THC and the density anomaly (4) to partially justify the approximation.

\subsection{Parameters}

We have reduced the problem to a pair of coupled equations (12)-(13) governing the thermal and saline perturbations in the cold box. The physics of the interactive THC illustrated in Fig. 2 enters mathematically through the terms that contain the inverse density-ratio $\gamma$. As such, it is seen to further differentiate the damping constants $\alpha_{1}$ and $\alpha_{2}$ while coupling the two equations through the coupling constants $c_{1}$ and $c_{2}$. In addition to the inverse densityratio $\gamma$, these constants contain two other dimensionless parameters: $\mu$ that measures the thermal damping due to the convective surface flux and $v$ that gauges the strength of the freshwater coupling. We shall now assess these dimensionless parameters in the observed system. 
The model time has been non-dimensionalized by the overturning time (10), which will first be estimated. Based on model calculations, we take the THC transport to be $20 \mathrm{~Sv}$ (Delworth and Greatbatch 2000; Biastoch et al. 2008). Dividing this by the surface area of the cold boxassumed spanning poleward of $40^{\circ} \mathrm{N}$ and comprising $1 / 6$ of the latitudinal circumference-yields $\bar{K} \approx 1.5 \times 10^{-6} \mathrm{~m} \cdot \mathrm{s}^{-1}$. Observation and model output suggest that the thermohaline anomalies associated with the AMV are confined to the upper several hundred meters of the convective region (Delworth et al. 1993; Hansen and Bezdek 1996), so we set $h \approx 500 \mathrm{~m}$. Dividing the two as in (10), the overturning time is $t_{o} \approx 10.6$ years or the time unit of the model is about a decade. For the stochastic surface heat flux, we set its standard deviation (SD) to be $10 \mathrm{~W} \mathrm{~m}^{-2}$ based on Delworth and Greatbatch (2000, their Fig. 8a). This corresponds incidentally to $1 \mathrm{~K}$ perturbation in the SAT if one applies the aerodynamics formula using a drag coefficient of $C_{d} \approx 10^{-3}$ and turbulent wind speed of $\left|u^{\prime}\right| \approx 10 \mathrm{~m} \cdot \mathrm{s}^{-1}$. With these values and setting $\Delta \bar{T}=20 \mathrm{~K}$, the dimensionless forcing $q$ ' of (11) has a SD of 0.08 .

Among the three dimensionless parameters, the inverse density-ratio $\gamma$ is subjected to direct measurement and different oceanic surveys have yielded rather consistent values to within $10 \%$ (Stommel and Csanady 1980; Tippins and Tomczak 2003), which is thus set at 0.35. With the above global temperature range, this inverse density-ratio implies $\Delta \bar{S}=1.5$, a reasonable range when compared with observations or model output (Peixoto and Oort 1992; Delworth et al. 1993). In contrast to the inverse densityratio, the other two dimensionless parameters ( $\mu$ and $v$ ) involve the ratio of fluxes hence are less certain and to somewhat allay the uncertainty, the solution will be examined over plausible ranges of these parameters.

To estimate $\mu$ from (18), we first used the above values of the drag coefficient and the turbulent wind speed to yield a transfer coefficient of $C \approx 2.38 \times 10^{-6} \mathrm{~m} \mathrm{~s}^{-1}$ from (2). The value of $\mu^{*}$ varies between 0 and 1 -depending on how strongly the SAT is linked to the SST, and there is a theoretical basis for a value of $1 / 2$ based on an entropy principle (Ozawa et al. 2003; Ou 2006), which is thus set as such as a reasonable value. Combined with the THC estimated above, we obtain $\mu \approx 0.8$. To estimate $v$ from (9), we first determine the peak SST and freshwater anomalies associated with the AMV. Taking the former to be $0.5 \mathrm{~K}$ (see Sect. 1), it would have a fractional value of 0.025 when normalized according to (5). For the freshwater flux from the Arctic Ocean, it has a climatological mean of $0.15 \mathrm{~Sv}$ and a peak anomaly of $0.024 \mathrm{~Sv}$, both based on Serreze et al. (2006, their Table 2). The bulk of the climatological freshwater input into the cold box $\left(\bar{F}_{w}\right)$ however, is due to the moisture convergence over the cold box (that is, the areal integral of P-E), which is difficult to estimate. Alternatively, based on time-mean balance of (3), it can be related to the salt flux associated with the THC or

$\bar{F}_{w}=\bar{K} \Delta \bar{S} / S_{o}$

and using the foregoing values and a reference salinity of 35 , we estimate $\bar{F}_{w} \approx 0.9 \mathrm{~Sv}$, so the above peak anomaly when normalized by this climatological mean is 0.026 . Dividing it by that of the SST, we obtain $v \approx 1.1$.The above discussion clearly underscores the high uncertainty in evaluating $\mu$ and $v$, both nonetheless are likely to be of $\mathrm{O}$ (1). In our discussion, we shall thus set $[\mu, v]=[1,1]$ as the standard case, and $[0,2]$ as both their plausible ranges over which the solution properties will be examined. For the standard case, the dimensionless parameters have the values of $c_{1} \approx 0.55, \quad c_{2} \approx 2.54, \quad \alpha_{1} \approx 3.54$ and $\alpha_{2} \approx 0.46$. It is seen in particular that partly because of the differential THC effect, the thermal damping constant $\left(\alpha_{1}\right)$ is much greater than its saline counterpart $\alpha_{2}$, and the corresponding damping timescales (their inverse multiplied by $2 \pi$ ) are 19 and 145 years, which thus differ by an order of magnitude, as alluded to before.

\subsection{Solution}

To simplify the notation, we define a bivariate vector $\vec{\varsigma}^{T} \equiv\left[T^{\prime} S^{\prime}\right]$, so the stochastic differential equations (12)(13) can be written in the matrix form:

$\dot{\varsigma}_{i}=-A_{i j} \varsigma_{j}+q_{i}^{\prime}$

adopting Einstein's convention of repetitive indices. In the above, the dynamical operator is

$A=\left[\begin{array}{cc}\alpha_{1} & -c_{1} \\ c_{2} & \alpha_{2}\end{array}\right]$,

and $q^{\prime}$ is assumed a Gaussian white noise of zero mean and covariance matrix

$\mathbf{q} \equiv\left[\begin{array}{ll}\varepsilon & 0 \\ 0 & 0\end{array}\right]$

with $\varepsilon$ being the forcing variance. The eigenvalues of the dynamical operator are given by

$\lambda_{1,2}=\frac{1}{\tau} \pm i \omega$

where

$\tau=2\left(\alpha_{1}+\alpha_{2}\right)^{-1}$,

and

$\left.\omega=\left[c_{1} c_{2}+\alpha_{1} \alpha_{2}-\left(\alpha_{1}+\alpha_{2}\right)^{2} / 4\right)\right]^{1 / 2}$ 
is the eigenfrequency; the eigenmode is oscillatory only if this eigenfrequency is real. The Green's functions of the dynamical operator are

$$
\begin{aligned}
G_{i j}(t) & =[\exp (-A t)]_{i j} \\
& =S_{0}(t) I_{i j}+S_{1}(t) A_{i j},
\end{aligned}
$$

with

$S_{0}(t)=\left(\lambda_{1}-\lambda_{2}\right)^{-1}\left[\lambda_{1} \exp \left(-\lambda_{2} t\right)-\lambda_{2} \exp \left(-\lambda_{1} t\right)\right]$,

and

$S_{1}(t)=\left(\lambda_{1}-\lambda_{2}\right)^{-1}\left[\exp \left(-\lambda_{1} t\right)-\exp \left(-\lambda_{2} t\right)\right]$,

and the solution to the stochastic differential equation (20) can be expressed in the Green's functions via (see Gardiner 1985)

$\varsigma(t)=G_{i j}(t) \varsigma_{j, 0}+\int_{0}^{t} G_{i j}\left(t^{\prime}\right) q_{j}^{\prime}\left(t-t^{\prime}\right) d t^{\prime}$,

where " 0 " in the subscript denotes the initial value. This solution will be used later to derive the time evolution of the forecast ensemble (Sect. 4).

\section{Genesis}

\subsection{Spectral properties}

To derive the spectral properties, we first solve for the Fourier transforms (overhats) from (20):

$\hat{\varsigma}_{i}(\sigma)=(A+i \sigma I)_{i j}^{-1} \hat{q}_{j}^{\prime}$,

with $\sigma$ being the Fourier frequency. Substituting (30) into the cross-spectral density defined by

$S_{i j} \equiv \hat{\varsigma}_{i} \hat{\varsigma}_{j}^{*}$

yields the spectral density of the thermal and saline fields (subscripted " $T$ " and " $S$ ", respectively):

$S_{T} \equiv S_{11}=2 \varepsilon\left(\alpha_{2}^{2}+\sigma^{2}\right) / D$,

and

$S_{S} \equiv S_{22}=2 \varepsilon c_{2}^{2} / D$

with $D \equiv\left(c_{1} c_{2}+\alpha_{1} \alpha_{2}-\sigma^{2}\right)^{2}+\sigma^{2}\left(\alpha_{1}+\alpha_{2}\right)^{2}$. From (8), the spectral density of the THC (subscripted " $K$ ") is then given by

$S_{K}=2 \varepsilon\left[\left(\alpha_{2}+\gamma c_{2}\right)^{2}+\sigma^{2}\right] /\left[(1-\gamma)^{2} D\right]$.

We have plotted in Fig. 3 the above spectral densities (normalized by the forcing variance $\varepsilon$ ) for the standard case, which show a broad thermal peak but essentially red

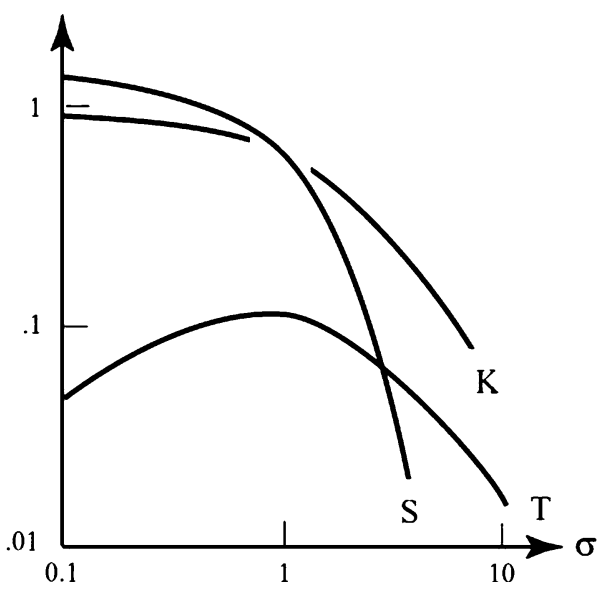

Fig. 3 The spectral densities of thermal (T), saline (S) and THC (K) perturbations, which have been normalized by the forcing variance and the Fourier period is in the unit of the overturning time. The saline and THC spectra are essentially red but the thermal spectrum exhibits a broad multidecadal peak

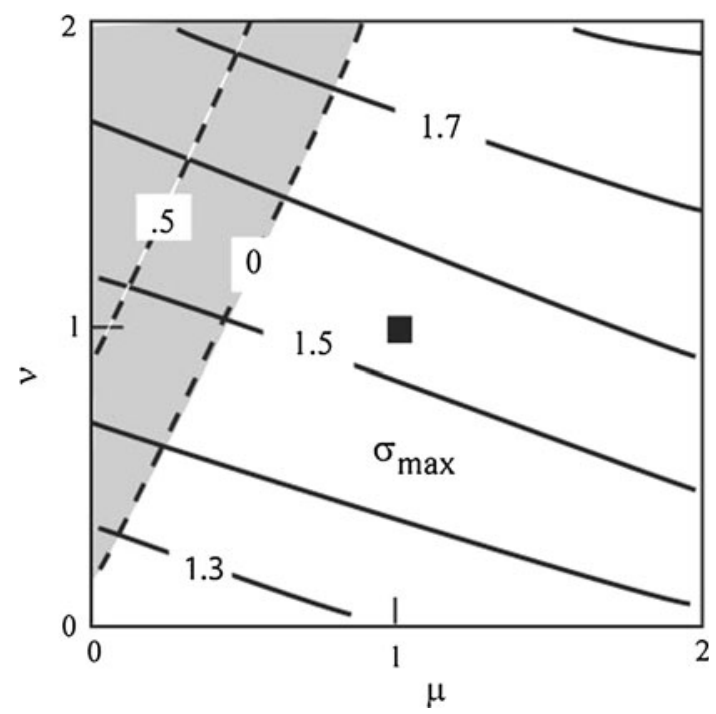

Fig. 4 The frequency of the thermal peak ( $\sigma_{\max }$, solid lines) and the eigenfreqency of the dynamical operator (dashed lines, nonzero only in the shaded region) plotted as functions of the thermal damping $(\mu)$ and freshwater coupling $(v)$ coefficients. The solid square marks the standard case, which is seen to contain no oscillatory mode, and the eigenfrequency, even when nonzero, bears no relation to the frequency of the thermal peak

saline and THC spectra. The frequency of the thermal peak is derived in Appendix A and plotted on the $\mu-v$ parameter space in Fig. 4 (the solid lines) with the solid square marking the standard case. It is seen that over this considerable parameter range, there is always a thermal peak, which is thus a robust feature of our model.

But as we shall demonstrate next, this thermal peak bears no relation to the oscillatory eigenmode of the deterministic dynamics. To see this, we have plotted the 
eigenfrequency (25) in the same figure (the dashed lines, real only in the shaded area). It is seen that the standard case contains no oscillatory mode, and even where an oscillatory mode does exist (in the upper-left shaded area), its frequency bears no relation to the frequency of the thermal peak; the latter thus may not be interpreted as a damped oscillation. But given the robustness of the thermal peak seen in the figure, it demands a simple explanation, which we shall advance next.

For instructive purpose, we first show in Fig. 5 the spectra (in dashed lines) when the saline effect on the thermal field is turned off (that is, $c_{1}=0$ ). Being an integration of the white-noise forcing by the ocean storage, the thermal spectrum is red but levels off beyond the thermal damping time (Hasselmann 1976), the latter marked by the shaded column on the right. Since the saline variance is induced by the thermal variance via the THC (13), it is doubly red and again levels off beyond the saline damping time (the shaded column on the left). Now we include the thermohaline coupling (that is, $c_{1} \neq 0$, the solid lines), then since it represents a negative feedback whose effect is proportional to the saline anomaly, it would more strongly suppress the low-frequency thermal variance (indicated by the arrow) to render a broad thermal peak bounded by the thermal and saline damping time. Conceptually, therefore, the thermal peak is not due to its

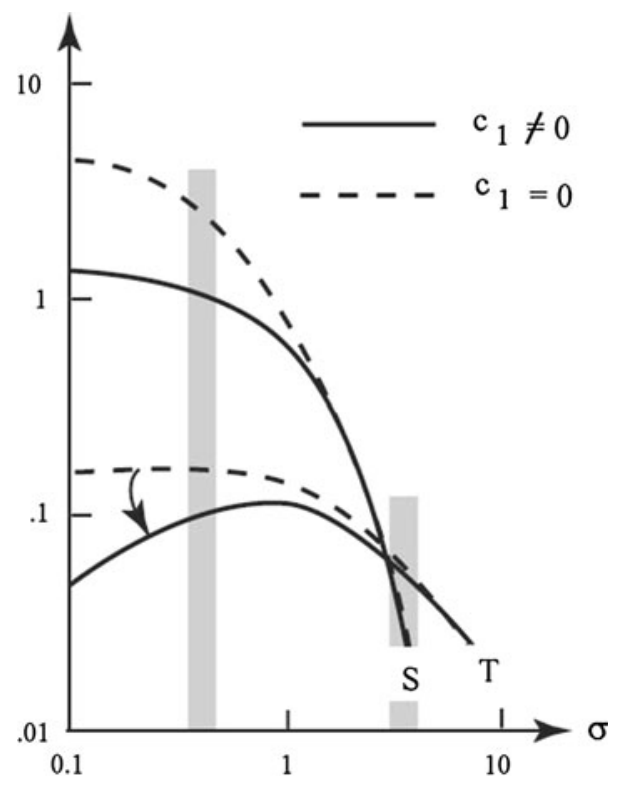

Fig. 5 Same as Fig. 3, but illustrating the effect of the thermohaline coupling. The dashed lines are when this coupling is artificially turned off by setting $c_{1}=0$, which show red thermal and saline spectra that level off at the thermal and the longer saline damping time (shaded columns), respectively. The thick lines are when the thermohaline coupling is turned on, which suppresses the low-frequency thermal variance (the arrow) to render a broad thermal peak bounded by the two damping times preferential amplification by the forcing, but rather the suppression of surrounding variance: the high-frequency side by the thermal inertia and the low-frequency side by the THC-induced negative feedback.

To further illustrate the robustness of the thermal peak, we define a "suppression" ratio as that of the coupled to the uncoupled thermal spectral-density at zero-frequency (the latter can be seen in Fig. 5 to approximate its peak value), which is given by (see [32])

Supp $=\left[1+\left(c_{1} c_{2}\right) /\left(\alpha_{1} \alpha_{2}\right)\right]^{-1}$.

Since all the dimensionless constants in (35) are positive, the suppression ratio is less than unity, as indeed seen in Fig. 6; there is thus always a thermal peak regardless the strength of the thermohaline coupling or damping. Qualitatively, stronger coupling $\left(c_{1}\right.$ and $\left.c_{2}\right)$ and weaker damping $\left(\alpha_{1}\right.$ and $\left.\alpha_{2}\right)$ imply smaller suppression ratio hence a more distinct thermal peak. Over the parameter range considered in Fig. 6, the suppression ratio is smaller than 0.5 , hence the thermal peak should be quite pronounced. With the above, we see that the negative feedback facilitated by the THC causes a robust thermal peak whose period is bounded by the thermal and saline damping time- the reason that it bears no relation to possible oscillatory modes.

Without the well-defined oscillation, one may no longer speak of its "amplitude", so to quantify the strength of the AMV, we calculate the standard deviation (SD) from the covariance derived in Appendix B and plot them in Fig. 7. Qualitatively, the thermal SD (Fig. 7a) decrease only slightly with $v$ as the low-frequency variance is further

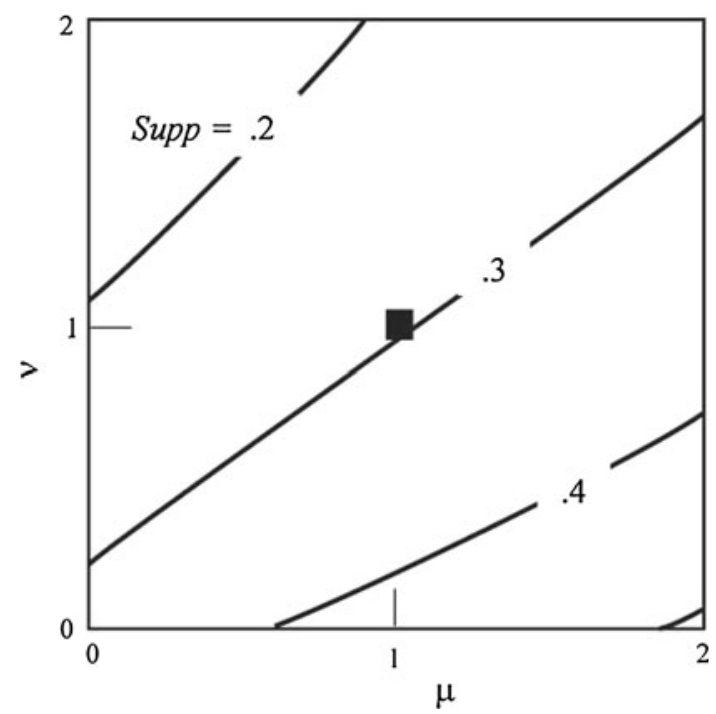

Fig. 6 Same as Fig. 4, but for the (suppression) ratio of the coupled to the uncoupled thermal spectral density at the zero-frequency, which measures the prominence of the spectral peak (more pronounced for smaller suppression ratio) 
Fig. 7 Same as Fig. 4 but for the standard deviation (SD) of the a thermal, b saline and c THC perturbations

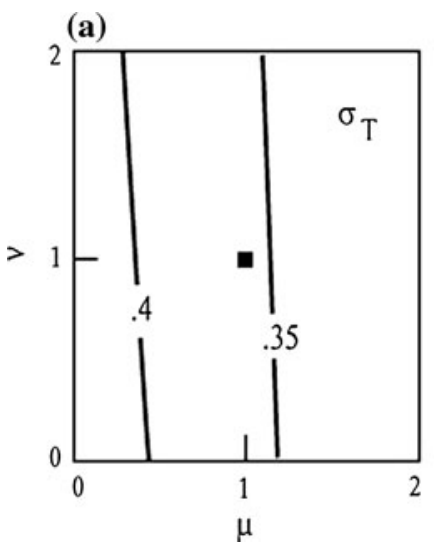

(b)

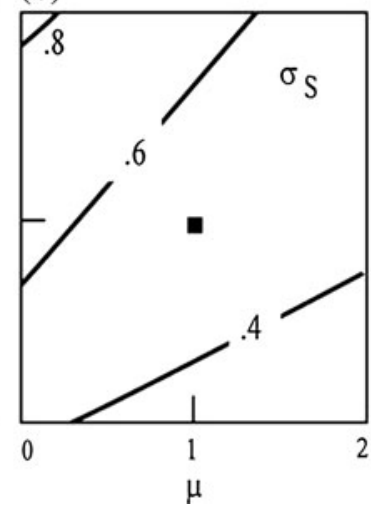

(c)

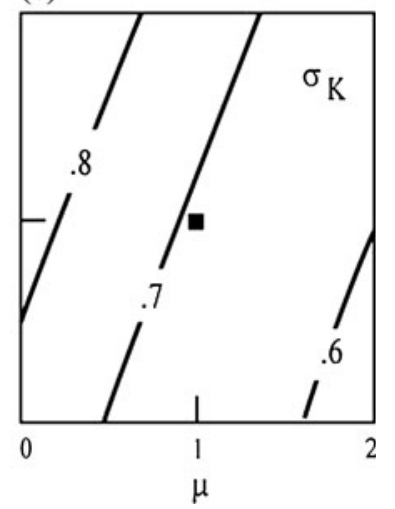

suppressed, but it decreases strongly with $\mu$ as the thermal damping is augmented. As the saline perturbation is induced by the thermal variance via the thermohaline coupling, its SD (Fig. 7b) decreases with $\mu$ that weakens the former, but increases with $v$ that augments the latter. With the saline SD varying more strongly over the parameter space than the thermal SD, it dominates the SD variation of the THC (Fig. 7c), which thus may be similarly explained. It is seen additionally that over the parameter range considered, the SD's of the thermal, saline and THC signals vary over a range of 30, 100 and 50\%, respectively, about their standard values.

\subsection{Observational comparison}

For the standard case, the timescale of the thermal peak is 43 years (Fig. 4), which fortuitously is the same as that calculated by Gray (2004) when averaged over five centuries of data (42.7 years). Although the model value varies only by $20 \%$ over the parameter range of Fig. 4 , its precise value has little significance given the broadness of the peak, which has as its tangible bounds only the thermal and saline damping time estimated earlier (Sect. 2.3) to be 19 and 145 years. This deduction in fact is consistent with the rather wide range gleaned from observational studies: Delworth and Mann (2000) calculated a thermal peak around 70 years from their three-century-long data; Gray et al.'s (2004)wavelet spectrum based on five-century-long data show a range between 40 and 128 years; and Stocker and Mysak (1992) discerned centennial peaks (90-140 years) from their millennium-long data-all within the bounds deduced above.

As symptomatic of the proposed genesis, only the thermal spectrum exhibits a prominent peak while the saline and the THC spectra are essentially red, as seen in Fig. 2. No comparable salinity spectrum as the thermal one is available, but sparse data show nonetheless sustained freshening of the subpolar oceans in the last four decades (Dickson et al. 2002; Curry and Mauritzen 2005), which seems to contrast the warm-cold cycle of the AMV and could be indicative of its redder spectrum-although the above-cited authors suggested that the freshening is due to the anthropogenic forcing rather than the natural variability.

There is of course no observed THC since it is merely a proxy mechanism for transporting the watermass properties that necessarily emerge when models do not fully resolve the ocean eddies (Sect. 2.1). Nonetheless, its spectrum based on the model output has been widely calculated-in fact more so than the thermal spectrum due perhaps to its easy indexing by a single number (the maximum transport) and also possibly to the preconception that an oscillatory AMV should manifest equally in the THC. Although these calculations have yielded possible peaks, they typically barely rise above the red background (Saravanan et al. 2000; Delworth and Greatbatch 2000; Holland et al. 2001; Knight et al. 2005; Danabasoglu 2008), which in fact is consistent with our model deduction. More telling, however, is when both THC and SST spectra are calculated and juxtaposed, such as in Timmermann et al. (1998), in which case thermal peak appears more pronounced and their contrast is not unlike that seen in Fig. 3.

To translate the SD's shown in Fig. 7 to dimensional units, we recall that they have been normalized by that of the fractional forcing $q^{\prime}(0.08)$ in addition to the scaling rules (5)-(7), so by combining the two, a unit SD in the thermal, saline and THC perturbation corresponds to dimensional values of $1.6 \mathrm{~K}, 0.12$ and $1.6 \mathrm{~Sv}$, respectively. Since for the standard case, the dimensionless SD's are $0.36,0.52$ and 0.69 for the above three fields (Fig. 7), the corresponding dimensional SD's are hence $0.6 \mathrm{~K}, 0.06$ and $1.1 \mathrm{~Sv}$, which can be compared with the observed or modeled variability. The peak SST anomaly of the AMV is about 0.5 K (Folland et al. 1984; Kushnir 1994; Kaplan et al. 1998; Hansen and Bezdek 1996; Gray 2004), and Latif et al. (2004) calculated a SD of $0.3 \mathrm{~K}$ from centurylong data, both thus commensurate with the deduced thermal SD. Dickson et al. (2002) showed a freshening of 


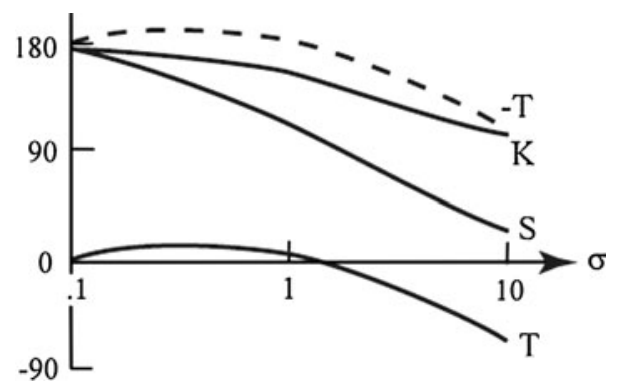

Fig. 8 Same as Fig. 3 but for the phase of the variables referenced to that of the forcing (positive for phase lead)

the subpolar water (the upper $1 \mathrm{~km}$ in correspondence to our cold box) of $\mathrm{O}(0.1)$ in the last few decades, similar to the range seen in Curry and Mauritzen (2005), hence not inconsistent with the deduced saline SD. The modeled THC associated with the AMV typically varies by no more than a few Sv (Delworth et al. 1993; Holland et al. 2001; Cheng et al. 2004; Dai et al. 2005; Jungclaus et al. 2005; Knight et al. 2005; Biastoch et al. 2008), and Latif et al. (2004) calculated a SD of $1.9 \mathrm{~Sv}$, hence they are commensurate with the deduced SD in the THC.

With the stochastic forcing hence its random phase, the lag of thermohaline anomalies to the forcing portends little significance, but the phase relation among the thermohaline perturbations themselves may nonetheless provide an additional test of the model physics. For this purpose, we plotted in Fig. 8 the phase of the thermohaline anomalies referenced to that of the forcing, which show that for interdecadal and lower-frequency bands, cold, salty and positive THC anomalies are all clustered within a quartercycle, with the positive THC slightly lagging the cold but leading the salty anomalies. This phase relation is consistent with that calculated by Delworth et al. (Delworth et al. 1993, their Fig. $8^{1}$ ), and the slight lag of the THC behind the density anomaly seen in their figure in fact supports the relation (4). It should be noted that only when the THC is an external forcing uncoupled to the thermohaline fields may one infer the lag of the latter owing to the ocean storage; the THC coupling (4) however, has supplanted this lag, so the effect of the ocean storage is simply to shift their collective phase with respect to the external (stochastic) forcing.

To recap, we see that although our model is extremely crude, its deductions compare nonetheless favorably - and in a quantitative sense-with observational data and output from climate models, suggesting that the model has captured the minimal physics of the AMV.

\footnotetext{
1 The thermohaline variables in this particular figure are averages over the sinking region hence more pertinent for comparison with our cold-box variables.
}

\section{Predictability}

With the above validation, we shall assume the model dynamics to be representative of the "true" dynamics of the AMV and assess its predictability if the forecasts are initialized with the "true" state. As both "true" qualifiers are at best approximate, the deduced predictability is an upper-bound or "potential" predictability, which will be gauged by metrics taken from the information theory.

\subsection{Metrics}

Since a forecast ensemble is defined by its probability distribution, we shall quantify the predictability by the departure of this distribution from that of the climatology (Schneider and Griffies 1999; Delsole 2004). A measure of such distribution difference is the relative entropy defined by (Cover 1991)

$R=\int_{-\infty}^{\infty} \int_{-\infty}^{\infty} p \ln \left(p / p_{\infty}\right) d \varsigma_{1} d \varsigma_{2}$

where $p$ is the forecast distribution and the subscript “ $\infty$ "denotes the climatology (corresponding to an infinite lead time). For a true initial state hence a delta-function distribution, the relative entropy is infinite, which can be shown to decrease monotonically for Markov processes and vanish if and only if the two distributions are identical (Cover 1991). For a stochastic forcing that is Gaussian, so are the transient distributions on account of the linear dynamics, which are thus uniquely specified by the ensemble mean and the covariance. Expressed in these properties, the relative entropy (36) can be divided into "dispersion" and "signal" components (Kleeman 2002):

$R=R_{d}+R_{s}$

with

$R_{d}=\frac{1}{2}\left[\ln \left(\left|V_{\infty}\right||V|^{-1}\right)+\operatorname{tr}\left(V V_{\infty}^{-1}\right)-2\right]$,

and

$R_{s}=\frac{1}{2}\langle\vec{\varsigma}\rangle^{T} V_{\infty}^{-1}\langle\vec{s}\rangle$,

in which angle brackets denote the ensemble means and $V$ 's are the covariance matrices. The two components are analogous to the root-mean-squared error and the anomaly correlation coefficient (Collins et al. 2006), but they are now combined into a single measure via their information content. We should point out the difference between the relative entropy and the predictive information (Schneider and Griffies 1999) as the latter is defined as a strict difference of the entropies hence contains no information on 
the ensemble means; but like the predictive information, the relative entropy is invariant to linear transformation of state variables, an important attribute since the information content should not depend on the units of the state variables-although in our formulation, the state variables, being the fractional perturbations, are already dimensionless.

The ensemble mean and the covariance that enter the relative entropy can be seen from (29) to be given by

$\left\langle\varsigma_{i}(t)\right\rangle=G_{i j}(t) \varsigma_{j, 0}$,

and

$V_{i j}(t) \equiv\left\langle\left(\varsigma_{i}-\left\langle\varsigma_{i}\right\rangle\right)\left(\varsigma_{j}-\left\langle\varsigma_{j}\right\rangle\right)\right\rangle=\int_{0}^{t} G_{i k}\left(t^{\prime}\right) G_{j l}\left(t^{\prime}\right) q_{k l} d t^{\prime}$.

Since the Green's functions depend only on the dynamical operator $\boldsymbol{A}$, the covariance matrix $V$ and hence the dispersion component $R_{d}$ are independent of the initial condition; its decrease in time thus is due solely to the error growth. On the other hand, the signal component is independent of the transient variance, and its decay in time is caused solely by the dynamical damping. With such differentiation of the two components, the optimal initial condition for the forecast is simply the one that maximizes the signal component.

To translate the infinite range in the relative entropy to a predictability measure, we define, following Schneider and Griffies (1999), the predictive power $P$

$P \equiv 1-\exp (-R)$

which is seen to conform to its intuitive meaning. That is, as the relative entropy decreases from infinity to zero for increasing lead time, the predictive power goes from unity (absolute predictability) to zero (no predictability). In addition, since the relative entropy decays monotonically with time, so does the predictive power of the full (bivariate) state; and to aid its rigor, Schneider and Griffies (1999) have provided a geometric interpretation of the predictive power on the state space.

Since the predictive power is a function of the lead time, a succinct measure of the predictability is the lead time when the predictive power falls below certain threshold. As this threshold depends on the accuracy one demands of the prediction or the odds one considers tolerable, there is obvious no set rules, and one often-used criterion in the univariate case is when the forecast variance reaches half the climatological variance whose tangible justification was provided by Griffies and Bryan (1997a), Scott (2003) and Chang et al. (2004). Applying (37) to the univariate case, the above criterion implies $R_{c}=0.097$ (subscript " $c$ " for "critical"), which is thus set for the general case. The corresponding predictive power (42) is $P_{c}=0.092$, which then defines the predictable time $\tau_{c}$.

The predictive power depends on the initial condition, which, being taken from observation, is itself subjected to climatological distribution, one thus may define an "average" (overhatted) predictive power as its expectant value for a forecast ensemble when its members are initialized by randomly chosen observed state, or

$$
\begin{aligned}
\hat{P} & \equiv \int_{-\infty}^{\infty} \int_{-\infty}^{\infty} p_{\infty} P d \varsigma_{1,0} d \varsigma_{2,0} \\
& =1-\exp \left(-R_{d}\right) \int_{-\infty}^{\infty} \int_{-\infty}^{\infty} p_{\infty} \exp \left(-R_{s}\right) d \varsigma_{1,0} d \varsigma_{2,0}
\end{aligned}
$$

taking note that the dispersion component $R_{d}$ is independent of the initial condition hence may be moved out of the integral. From (39) and (40), it is seen that $R_{s}$ is quadratic in the initial anomaly, so is the exponent in the climatological distribution; an analytical expression thus may be derived for (43) as given in Appendix C. Applying the same threshold criterion, one may define the "average" predictable time $\hat{\tau}_{c}$, which is then a property only of the dynamics, independent of the initial condition.

All above metrics apply to the full bivariate state, but for practical reasons, one might be more concerned with the predictability of individual variables. This would be the case for the AMV, which is defined by the SST anomaly, and the latter moreover directly impacts the surface climate hence is of greater practical concern. The univariate version of the relative entropy is called the "marginal" entropy, whose expression is given in Appendix C, and using the marginal entropy, the predictive power and predictable time for the individual variables can be similarly defined. In contrast to the relative entropy, however, which decays monotonically, marginal entropy may temporarily increase since information can be transferred among state variables. As such, there could be a return of skill in forecasting individual variables (Barlas et al. 2007) and the predictable time based on a threshold condition could be multi-valued.

The following specific calculations of the predictability pertain to the standard case, and we will discuss its parameter dependence in Sect. 4.4.

\subsection{Forecast ensemble}

In Fig. 9, we show the evolution of the forecast ensemble on the state space for the three cases marked by the open squares (enclosing the case number). To aid their selection, we have plotted the climatological ellipses of 1,2 and $3-\mathrm{SD}$, and the outlying cases are the intersections of the 3-SD ellipse with a vertical line (dashed) that is tangent to 


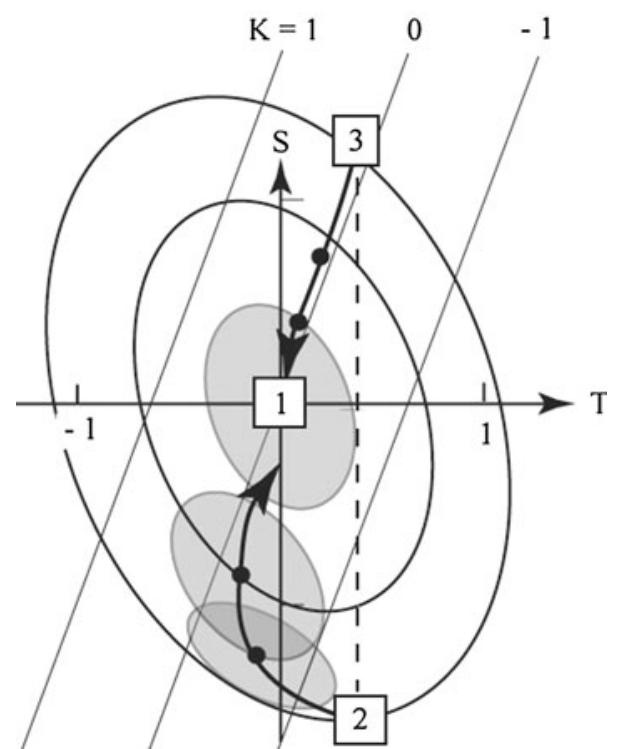

Fig. 9 The evolution of the forecast ensemble on the state space, on which the climatological ellipses of 1, 2 and 3-SD (concentric about the origin) and constant THC (light straight lines) are drawn. The three initial states (cases 1,2 and 3) are marked by open squares with the arrowed lines tracing the ensemble mean (solid circles in 0.5 time units) and 1-SD forecast ellipses shown along one track

the 1-SD ellipse. As such, they have the same thermal anomaly (0.35) but saline anomalies that are of opposite signs ( -1.55 for case 2 and 1.29 for case 3 ) and, being on the same ellipse, they have equal signal components (hence relative entropy) initially, which would facilitate their predictability comparison. By integrating the bivariate normal distribution over the probability ellipse, it's straightforward to derive that the exceedance probability (or complementary cumulative probability) for the three ellipses has the value of $0.61,0.14$ and .01, respectively, so the events outside the 3-SD ellipse for example have less than $1 \%$ chance of occurrence.
The evolution of the ensemble means is indicated by arrows with the solid circles marking 0.5 in time increments. Because of the strong damping, the outlying cases decay to climatology rapidly though remnant oscillation is discernible for case 2 (that is, the thermal anomaly has changed sign). Case 3 approaches the climatology at a faster rate than case 2 due to the thermohaline coupling: the positive thermal anomaly tends to induce negative salinity anomaly (13), which thus counters the decay of the saline anomaly in case 2, but hasten it in case 3 .

Since the covariance is independent of the initial condition, it is displayed along track 2 only via the 1-SD ellipses. We see pronounced anisotropy in the short-term error growth, being much faster for the thermal than the saline fields. This is because the stochastic forcing is in the surface heat flux hence only directly generates the thermal variance whereas the saline variance is induced by the thermal variance through the latter's effect on the THC (13). Indeed, one can see from (41) that the growth rate of the thermal variance equals the forcing variance initially (and importantly it is independent of the dynamics) while that of the saline variance is initially zero and accelerates for short lead-time. Combined with the negative correlation between the thermal and saline perturbations discernible from (13), one may explain the clockwise rotation of the ellipses. One recognizes that while the ellipse rotation stems from the non-normal dynamics, it is the negative thermohaline feedback that endows this specific sense of rotation. It is seen that within unit lead-time, the error ellipse has largely attained its climatological size, beyond which it is primarily the ensemble mean that may sustain the predictability, as discussed next.

We have plotted the predictive power for the above selected cases in Fig. 10, with the dashed line marking the threshold that defines our predictable time (Sect. 4.1). Most relevant to the surface climate is the univariate thermal
Fig. 10 The predictive power of the three selected cases for a thermal, b saline, c THC and d bivariate $\mathrm{T} / \mathrm{S}$ signals
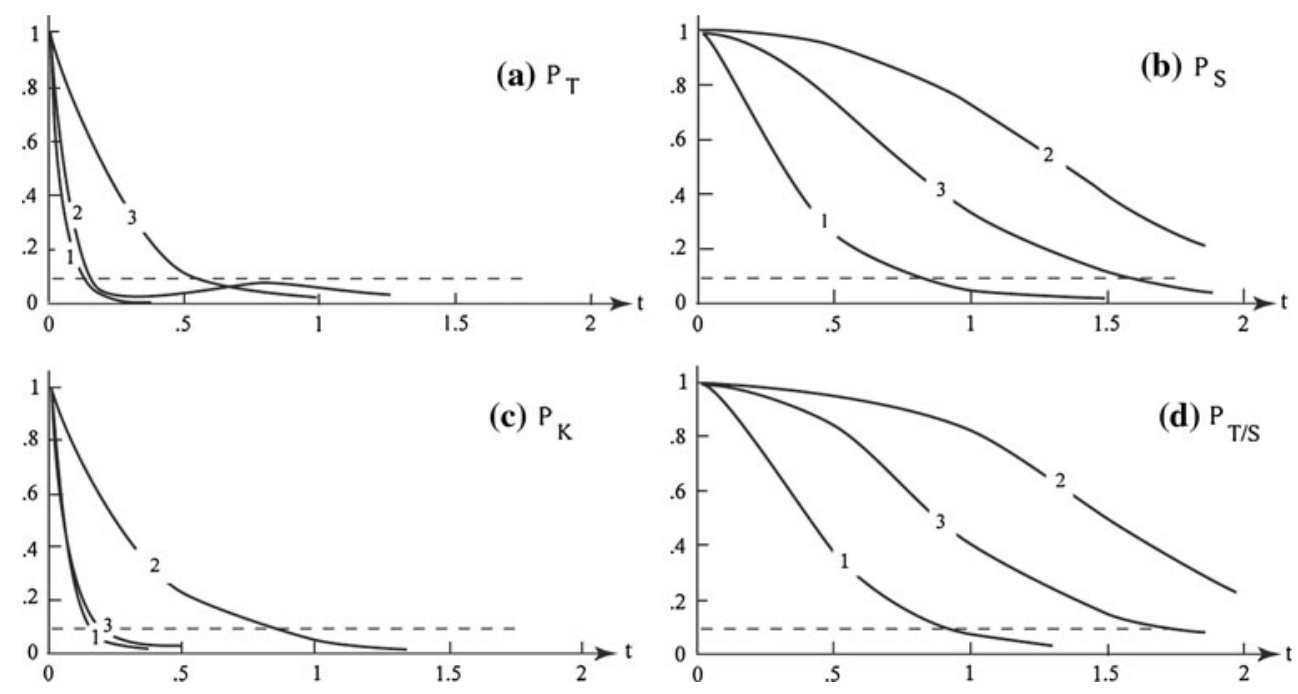
curves shown in Fig. 10a. For case 1 initialized by the climatology, the loss of the predictive power is due solely to the growth of the thermal noise, which has a unity initial rate (normalized by the forcing variance), but decreases gradually over the short lead-time. As such, it is the small climatological variance due to the strong thermal damping that yields the short predictable time (about 0.14). For case 2 , the addition of the signal component only slightly prolongs the predictability due to the strong thermal decay seen in Fig. 9, which has been hastened by the negative saline anomaly (12). Also expected from that figure, we notice a slight return of forecast skill indicative of the remnant oscillation, which, however, is too small to have practical significance. For case 3, the positive saline anomaly has slowed the thermal decay (12) to more strongly enhance the predictability, lengthening the predictable time to 0.5 .

The predictive power for the saline field is as shown in Fig. 10b, which differs sharply from its thermal counterpart. The convex shape of the curves over the short leadtime reflects the error growth that is zero initially but accelerates over the short lead-time. In comparison with the thermal curves, the predictive power is strongly augmented in the outlying cases, first by the slower error growth, then by the weak saline damping. The differentiation between the two outlying cases is as seen in Fig. 9, which is attributable to the thermal effect: namely, the positive thermal anomaly tends to induce negative salinity (13), which thus counters the saline decay in case 2, but hastens it in case 3 . The saline predictable time is several times the corresponding thermal time.

For the THC, we see that its predictive power is similar to the thermal curve for case 1 . This is because, as one may see from (8), its short-term error growth is dominated by the much faster thermal than the saline rate. Since its signal component is near zero for case 3 (see Fig. 9, the thin straight lines), it has scarcely modified the predictive power of case 1 , but for case 2 , the sizable THC anomaly has significantly augmented the predictive power via the signal component. The latter incidentally is consistent with the finding of the Collins et al. (2006). Since the THC is linked to the anomalous density, it has the same predictability as the dynamical height, the latter has been considered by Griffies and Bryan (1997b).

Since the relative entropy is greater than the marginal entropy - the latter contains only partial information, so is the bivariate predictive power in comparison with its univariate counterparts. Considering also the dominance of the saline over the thermal predictive power, the full curves should be similar to-but slightly above-the corresponding saline curves, as indeed seen in Fig. 10b and d: the predictability of the bivariate state is thus similar to that of the salinity field. Since the relative entropy is monotonically decreasing, so should the bivariate predictive power, one infers therefore that there is not likely any return of skill in forecasting the salinity anomaly, in contrast to the thermal anomaly.

To recap, from the evolution of the forecast ensemble on the state space, we differentiate the roles of the error growth and dynamical damping in the loss of the predictive power. Both are highly anisotropic: the growth of the thermal noise is much faster due to its direct generation by the stochastic forcing; and the decay of the thermohaline signal is first differentiated by the THC and then by the initial condition. The fast growth of the thermal noise strongly curtails the thermal predictability while the saline predictive power is prolonged by both the slower error growth and weaker damping. Since the short-term THC noise is dominated by the thermal noise, the two have similar predictability; and with the bivariate predictive power being a cap to its univariate counterparts, it is dominated by-hence similar to-its saline counterpart.

\subsection{Optimization}

Some optimizing measures of the predictability have no direct applications to the current problem. For example, it is the nature of the stochastic forcing that it enters mainly through the surface heat flux (Sect. 2.1); there are thus no degrees of freedom associated with a stochastic optimal (Kleeman and Moore 1997; Tziperman and Ioannou 2002). Since the AMV is defined by the SST, which moreover directly impacts the surface climate, it serves little practical purpose in determining the optimizing predictable components (DelSole 2007). The optimization that can be readily exploited in the AMV prediction is the initial anomaly vector, and to quantify its effect, we plot in Fig. 11 the predictable time as a function of the initial condition spanning the state space. The dots indicate explicit calculations used to draw the contours: they are intersections of the outer ellipses (and the T-axis) with the vertical lines that glaze the inner ellipses. The contours are shown only for the half plane on account of the central symmetry, and minimum predictable time is shaded to aid the display.

The predictable time of the thermal field is shown in Fig. 11a, which lengthens with initial departure from the climatology (hence the signal component), but the effect is highly anisotropic, being much greater when the saline and thermal anomalies are of the same sign, as explained earlier (Sect. 4.2). Case 3 incidentally represents the optimal condition on its ellipse: its predictable time is about four times the minimum (0.6 vs. 0.15). Based on the scales estimated in Sect. 3.3, case 3 has a thermal anomaly of $0.57 \mathrm{~K}$ and saline anomaly of 0.16 . While these values are not out of reach, they are expectedly rare since they lie on 

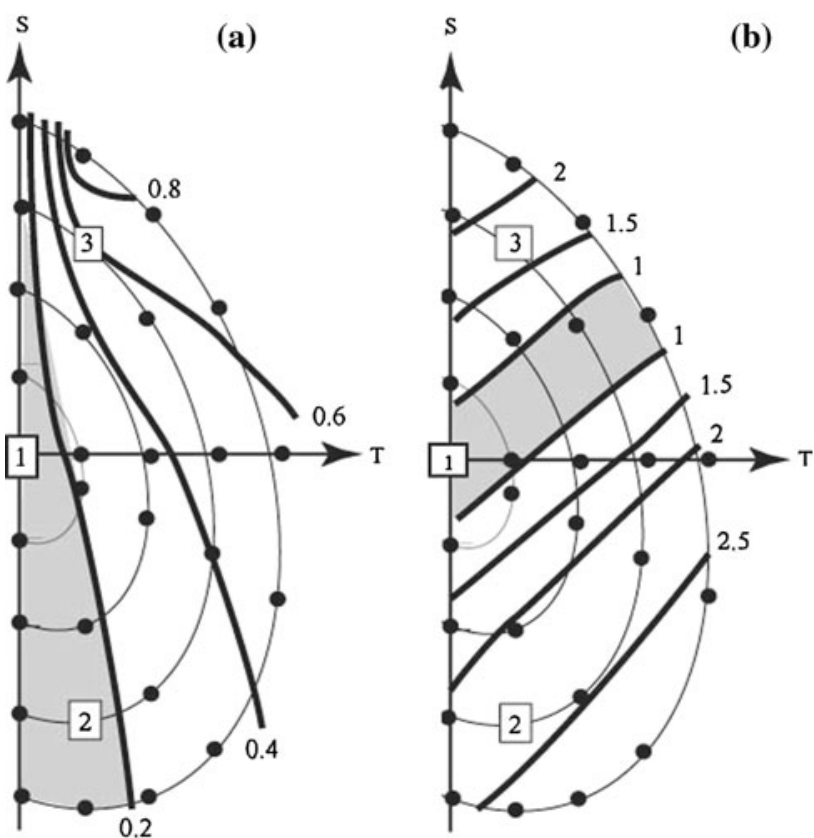

the cool period of the 1980s (Talley and McCartney 1982), a concurrence that also manifests in model calculations (Delworth et al. 1997), which may aid the optimization. But even with these favorable conditions, the predictable time is seen in Fig. 11a to fall short of a decade, and given the multidecadal timescale that characterizes such major events, there is practically no skill in predicting their next arrival. As a related point, this deduction further underscores the vast difference of the AMV from an oscillatory mode, the latter, being predicated on weak damping, is naturally more predictable; and to the degree that ensemble experiments generally yield similar predictable time as the above estimate (Griffies and Bryan 1997b; Grotzner et al. 1999; Saravanan et al. 2000; Eden 2002; Collins et al. 2006; Tziperman et al. 2008), which moreover is commensurate with the thermal damping time, they in fact support a non-oscillatory nature of the AMV.

The predictable time for the salinity signal is shown in Fig. 11b. If without its coupling to the thermal field, the contours would be horizontal and their value increases away from the T-axis, but as discussed in Sect. 4.2, the thermal effect would lengthen (shorten) the predictable time if the saline anomaly is of the opposite (same) sign, resulting in a counterclockwise rotation of the contours. The predictable time for the THC is plotted in Fig. 11c, which conspicuously align with the THC lines shown in Fig. 9 hence may be subjected to the following interpretation: the THC error growth is dominated by the thermal noise (8), which accounts for their similarly short minimum time of $\mathrm{O}(0.1)$, and beyond this time, its predictive power is sustained mainly by the signal component hence aligned with the initial THC anomaly. The predictable time for the bivariate state is shown in Fig. 11d, which, based on the earlier discussion, should resemble but cap the saline values.

\subsection{Parameter dependence}

Having considered the effect of the initial condition on the predictability, we now consider the predictive power averaged over the initial condition as defined in Sect. 4.1. It represents the expectant predictability when the ensemble members are initialized by randomly chosen observed state. We show in Fig. 12 the average predictive power. When compared with Fig. 10, it is naturally straddled by the respective curves, but it is nonetheless closer to the null curve (case 1) as the climatological mean obviously has the highest probability hence most heavily weighted. As a general characterization, the predictive powers fall into two distinct groups: the group of thermal and THC signals is much less predictable than the group of saline and bivariate signals. The average predictable time is 0.22 and 0.36 for the first group and 1.35 and 1.49 for the second, which can 


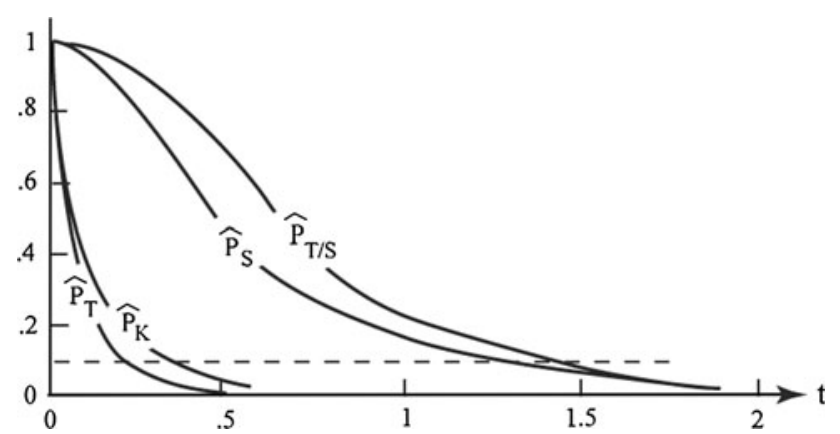

Fig. 12 The average predictive power when the initial condition is weighted by the climatological distribution

be converted to dimensional units by multiplying them by a decade (Sect. 2.3). We see therefore the average predictable time for the thermal anomaly is only a couple of years while that for the saline anomaly may exceed a decade- $-\mathrm{a}$ disparity not inconsistent with that found in some model studies (for example, Griffies and Bryan 1997a).

But how robust is the average predictability? How does it vary with the model parameters? To address these questions, we plot in Fig. 13 the average predictable time over the parameter range considered in Sect. 3. Since the average
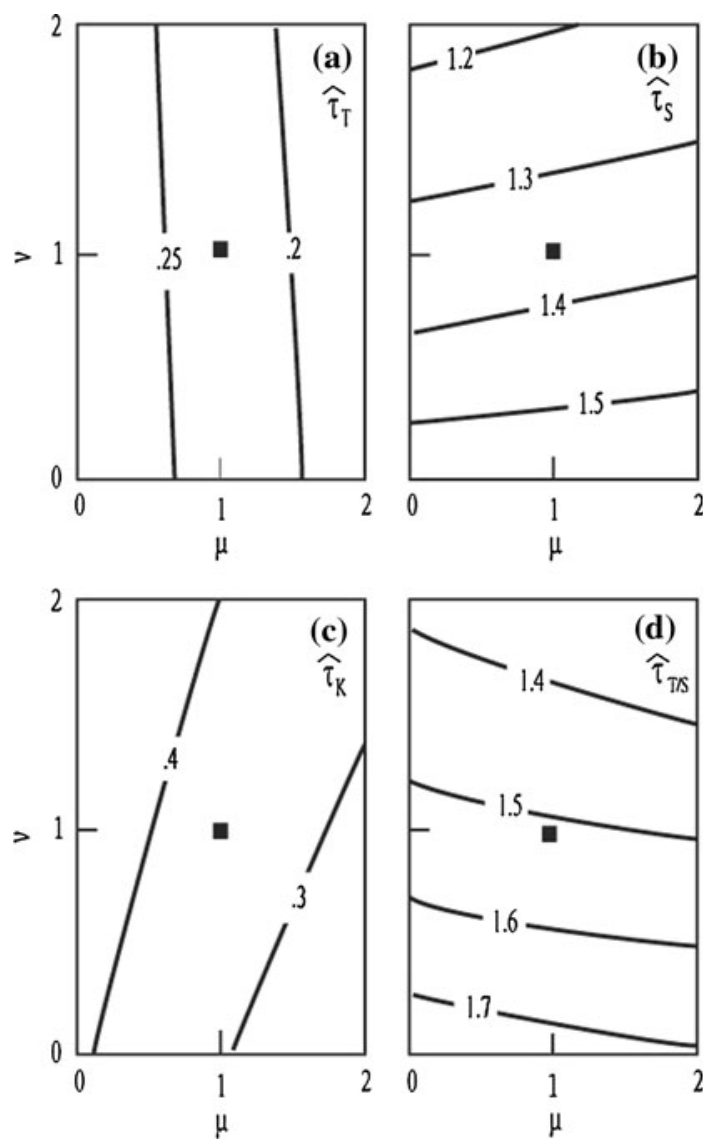

Fig. 13 The average predictable time for a thermal, b saline, c THC and $\mathbf{d}$ bivariate $\mathrm{T} / \mathrm{S}$ perturbations predictability is noted above to be similar to that for the null case, its parameter dependence can be explained by the dispersion component alone, as provided below.

For the thermal anomaly, we note immediately that its dependence is similar to that of the climatological variance shown in Fig. 7a, which can be attributed to the fact that the short-term error growth rate is approximately unity, independent of the dynamics. Since the predictable time for the null case is when the error growth attains half the climatological variance, by simply dividing the two, we obtain a predictable time of 0.07 for the standard case. This obviously constitutes a lower bound for the average predictable time since it needs to be adjusted upward due to the additional information contained in the initial anomaly and the fact that the error growth slows in time. These effects have apparently lengthened the predictable time about threefold to 0.23 as seen in Fig. 13a.

Since the climatological variance equals the integral of the spectral density on account of the Parseval's theorem, it is seen that the spectral shape, including possible peaks, has no direct bearing on the predictability. As a counter example, we see from Figs. 6 and 13a that increasing the freshwater coupling would render a more distinct thermal peak, and yet by reducing the thermal variance, it actually weakens the predictability. On the other hand, if a sharper spectral peak is the outcome of weaker damping (moving to the left in these figures), then the latter's augmentation of the climatological variance would prolong the predictability. The important point, however, is that there is no causal linkage between the spectral peak and predictability (see also Chang et al. 2004), but rather, both respond in kind to the damping.

The predictable time for the saline signal (Fig. 13b) is several times longer than its thermal counterpart and also exhibits qualitatively different behavior. It shortens with stronger freshwater coupling but is insensitive to the thermal damping, and importantly, unlike the thermal time, it does not vary as the climatological variance shown in Fig. $7 \mathrm{~b}$. This is because the growth of the saline noise, unlike that of the thermal noise, can be hastened by the freshwater coupling (13), an effect that apparently dominates the variation of the climatological variance in controlling the predictable time.

For the THC, its average predictable time (Fig. 13c) is only slightly longer than the thermal time, and again aligned with its climatological variance, hence may be interpreted similarly. The predictable time for the bivariate $\mathrm{T} / \mathrm{S}$ is shown in Fig. 13d, which varies similarly as the saline time, at least in terms of the dominant effect of the freshwater coupling. It does have minor dependence on the thermal damping, which is opposite to that for the saline field and can be explained by the effect of the thermal predictable time. 
With the above, it is seen that over the plausible parameter range, the average predictable time does not vary by more than $50 \%$ from the standard case, we conclude therefore, as a general characterization, the average predictable time for the thermal and the THC signal is well short of a decade while that for the saline and the bivariate field is decadal. Since AMV is defined by the thermal anomaly, we conclude therefore it is largely unpredictable.

\section{Summery and discussion}

The AMV is dominated by the SST anomaly in the subpolar North Atlantic, which exhibits discernible multidecadal timescale. Assuming that the AMV is a small perturbation of the base state sustained by the stochastic atmosphere, we construct a box model to examine its genesis and predictability. Through thermohaline balances of the subpolar cold box coupled through a densitydependent THC, it is seen that the latter would strongly differentiate the thermal and saline damping, and facilitate a negative feedback between the two fields. This negative feedback would preferentially suppress the low-frequency thermal variance, and together with its high-frequency suppression by the ocean storage, the thermal spectrum exhibits a broad peak bounded by the thermal and saline damping time. We offer this "differential variance suppression" as an alternative paradigm of the AMV in place of the prevailing "damped oscillation"-as the latter is generally not allowed by the deterministic dynamics and in any event bears no relation to the thermal peak. Although the model is extremely crude, its deductions compare favorably-and in quantitative sense-with the observed variability, suggesting that the model has captured the minimal physics of the AMV.

With validation of the model dynamics, we then consider the potential predictability of the AMV based on the relative entropy - a measure of the difference between the forecast and climatological (probability) distributions, which may decay due both to the error growth and dynamical damping of the ensemble mean. Since the stochastic forcing is mainly in the surface heat flux, the thermal noise grows rapidly and together with a climatological variance limited by the THC-enhanced thermal damping, they strongly curb the thermal predictability. The predictability can be optimized by initial thermal and saline anomalies that are of the same sign, but even for rare events of less than $1 \%$ chance of occurrence (for example, a decade-long anomaly that occurs only once in a millennium), the predictable time still falls well short of a decade; we contend therefore that the AMV defined by the thermal anomaly is effectively unpredictable. Due both to slower error growth and weaker damping, the saline anomaly, however, has longer predictable time, which may exceed a decade.

Within the proposed paradigm, some outstanding questions of the AMV may be resolved. For example, the THC spectra are widely calculated in modeling studies, which typically show unremarkable peaks that barely rise above the red background. This, however, is to be expected since the low-frequency suppression of the thermal variance is due precisely to the redder saline and-hence-THC spectra. The absence of the THC peak, however, should not equivocate the robustness of the thermal peak that defines the AMV, as seemingly suggested by some model studies. Since the thermal peak is unrelated to oscillation and bounded only broadly by the disparate thermal and saline damping time (decadal versus centennial), the model may explain the observed migratory behavior of the thermal peak within this range (Gray 2004) and equally divergent timescales produced in climate models (Delworth et al. 2007; Danabasoglu 2008).

Model runs with only stochastic freshwater forcingthough of little relevance to the AMV-have produced spectral peak of much longer period (200-300 years, Mikolajewicz and Maier-Reimer 1990; Mysak et al. 1993), which can be accommodated within the proposed paradigm. Since the ocean dynamics hence the eigenmodes are unchanged by the external forcing, this disparate timescale from that of the AMV further discounts the relevance of the eigenmodes to the spectral peak. With the absence of the thermal forcing and the strong thermal damping, the thermal spectrum, however, should mimic the saline spectrum; and following the same reasoning as that for Fig. 5, the saline spectrum should be red and level off beyond the saline damping time (about 150 years) - if without the THC coupling, but with the low-frequency suppressed of its variance by this coupling, the saline and-hence-all spectra should exhibit a spectral peak beyond the saline damping time, as seen in the above model runs.

There have been discussions as to whether the AMV is a coupled or ocean-only mode, but based on our paradigm, it's first of all not a mode, but is the remnant when the surrounding variance is preferentially suppressed. The source of the variance is the stochastic atmosphere, which is uncoupled to the climatic SST, and the processes that differentially suppress the variance are oceanic-thermal inertia on the high-frequency side and THC-induced negative feedback on the low-frequency side. The moisture coupling considered by Timmermann et al. (1998) is contained in $v$, which in fact is dominated by the Arctic effect (Sect. 2.3), but nonetheless since $v$ merely augments the THC effect (15), it is not required for the positivity of the coupling constant $c_{2}$ and hence the genesis of the AMV. With the above, our view thus is closer to Delworth and Greatbatch's (2000) that the AMV is generically an 
oceanic phenomenon. In addition, with the Arctic dominating the freshwater coupling, our model may explain why climate models that include such coupling tend to produce more pronounced AMV (Delworth et al. 1997).

While the atmospheric coupling is not required for the thermal peak, it does affect the height of the peak through $\mu$ in (16): a stronger thermal coupling implies a smaller $\mu$ hence weaker damping, resulting in a stronger AMV signal. This could explain why in coupled models, the surface heat flux may attain greater anomaly particularly in the convective region (Delworth and Greatbatch 2000) despite its amelioration by a responsive SAT (Barsugli and Battisti 1998). Moreover, because of the atmospheric coupling, the observed SAT spectrum contains the imprint of the SST variability hence may not be taken as the external forcing-as implicated in the use of restoring condition in ocean-only models. Rather, to isolate the external forcing, we have invoked the output from atmosphere-only models that have produced the stochastic hence uncoupled forcing (Sect. 2.3). With the latter now justifiably posed as the external forcing and together with the inner working of the ocean that differentiates its frequency response, the causality of the AMV thus may be established.

The THC in the model represents a proxy of the meridional transport mechanism of watermass properties, which may be facilitated as well by eddy exchanges. In fact, recent observational analysis suggest that eddies dominate the THC, and there is little evidence of a contiguous MOC depicted in climate models (Lozier 2010). Since in such models, MOC is the ageostrophic flow driven by the meridional pressure gradient and with fixed diapycnal diffusivity, its transport is expectedly proportional to the density contrast, as postulated in our model. But if the THC is dominated by random eddy exchange, can we still justify this linkage? One possible argument in its favor goes as follows: if the exchange were random, then its rate is independent of the density stratification; the latter, however, does govern the eddy dimension through the deformation radius. Since the volume exchange rate (the relevant quantity that corresponds to the MOC transport) is the product of the eddy exchange rate and the eddy volume, it is then proportional to the density stratification, as required. Here we reiterate the advantage of using the fractional perturbations so that the solution is independent of the proportional constant-hence the unknown eddy exchange rate.

Acknowledgments Despite several attempts, the work has not received research grants. I want to thank Drs. Michael Purdy and Arnold Gordon for their support, anonymous reviewers for their insightful comments and my colleagues at Lamont for stimulating discussions through the years.

\section{Appendix A: Spectral peak}

Differentiating the thermal spectral density (32) with respect to the Fourier frequency and setting it to zero, we derive the spectral peak at

$\sigma_{\max }=\left(-\alpha_{2}^{2}+H^{1 / 2}\right)^{1 / 2}$

where

$H \equiv \alpha_{2}^{4}+2 \alpha_{2}^{2}\left(d_{2}-d_{1}^{2} / 2\right)+d_{2}^{2}$,

with

$d_{1}=\alpha_{1}+\alpha_{2}$,

$d_{2}=c_{1} c_{2}+\alpha_{1} \alpha_{2}$.

\section{Appendix B: Covariance}

From (41), we derive that

$$
\begin{aligned}
& V_{11}=\varepsilon \int_{0}^{t} G_{11}\left(t^{\prime}\right)^{2} d t^{\prime}=\frac{\varepsilon}{2\left(\lambda_{1}-\lambda_{2}\right)^{2}} \\
& \left\{\frac{\left(\lambda_{1}-\alpha_{2}\right)^{2}}{\lambda_{1}} \cdot\left(1-e^{-2 \lambda_{1} t}\right)+\frac{\left(\lambda_{2}-\alpha_{2}\right)^{2}}{\lambda_{2}} \cdot\left(1-e^{-2 \lambda_{2} t}\right)-\right. \\
& \left.\frac{4\left(\lambda_{1}-\alpha_{2}\right)\left(\lambda_{2}-\alpha_{2}\right)}{\lambda_{1}+\lambda_{2}} \cdot\left(1-e^{-\left(\lambda_{1}+\lambda_{2}\right) t}\right)\right\}, \\
& V_{22}=\varepsilon \int_{0}^{t} G_{21}\left(t^{\prime}\right)^{2} d t^{\prime}=\frac{\varepsilon c_{2}^{2}}{2\left(\lambda_{1}-\lambda_{2}\right)^{2}} \cdot\left\{\frac{1}{\lambda_{1}} \cdot\left(1-e^{-2 \lambda_{1} t}\right)\right. \\
& \left.+\frac{1}{\lambda_{2}} \cdot\left(1-e^{-2 \lambda_{2} t}\right)-\frac{4}{\lambda_{1}+\lambda_{2}} \cdot\left(1-e^{-\left(\lambda_{1}+\lambda_{2}\right) t}\right)\right\}, \\
& V_{12}=\varepsilon \int_{0}^{t} G_{11}\left(t^{\prime}\right) G_{21}\left(t^{\prime}\right) d t^{\prime}=\frac{\varepsilon c_{2}}{2\left(\lambda_{1}-\lambda_{2}\right)^{2}} \cdot\left\{\frac{\left(\lambda_{1}-\alpha_{2}\right)}{\lambda_{1}}\right. \\
& \cdot\left(1-e^{-2 \lambda_{1} t}\right)+\frac{\left(\lambda_{2}-\alpha_{2}\right)}{\lambda_{2}} \cdot\left(1-e^{-2 \lambda_{2} t}\right)- \\
& \left.\frac{2\left[\left(\lambda_{1}-\alpha_{2}\right)+\left(\lambda_{2}-\alpha_{2}\right)\right]}{\lambda_{1}+\lambda_{2}} \cdot\left(1-e^{-\left(\lambda_{1}+\lambda_{2}\right) t}\right)\right\} .
\end{aligned}
$$

Setting $t \rightarrow \infty$, we obtain the climatological covariance:

$$
\begin{aligned}
& V_{11, \infty}=\varepsilon \cdot \frac{\left(c_{1} c_{2}+\alpha_{1} \alpha_{2}+\alpha_{2}^{2}\right)}{2\left(c_{1} c_{2}+\alpha_{1} \alpha_{2}\right)\left(\alpha_{1}+\alpha_{2}\right)}, \\
& V_{22, \infty}=\varepsilon \cdot \frac{c_{2}^{2}}{2\left(c_{1} c_{2}+\alpha_{1} \alpha_{2}\right)\left(\alpha_{1}+\alpha_{2}\right)},
\end{aligned}
$$


$V_{12, \infty}=-\varepsilon \cdot \frac{c_{2} \alpha_{2}}{2\left(c_{1} c_{2}+\alpha_{1} \alpha_{2}\right)\left(\alpha_{1}+\alpha_{2}\right)}$

Tagging the THC as the variable " 3 ", its variance can be derived from (8) to yield

$V_{33}=\frac{1}{(1-\gamma)^{2}} \cdot\left(V_{11}-2 \gamma V_{12}+\gamma^{2} V_{22}\right)$.

The standard deviation $(\sigma)$ is the square root of the autovariance hence can be calculated.

\section{Appendix C: Average predictive power}

The average predictive power is given by (8) with

$p_{\infty}=\frac{1}{2 \pi \sqrt{\left|V_{\infty}\right|}} \exp \left(\frac{1}{2} \vec{\varsigma}_{0}^{T} V_{\infty}^{-1} \vec{\varsigma}_{0}\right)$.

Substituting from (39) to (40) the signal component of the relative entropy, (4.8) can be integrated to yield

$\hat{P}=1-\exp \left(-R_{d}\right) \cdot \frac{1}{2}\left(\left|V_{\infty}\right| a d\right)^{1 / 2}$,

in which the temporal functions are given by

$d=c-b^{2} /(4 a)$,

with

$a=\frac{1}{2\left|V_{\infty}\right|}\left(V_{22, \infty} G_{11}^{2}-2 V_{12, \infty} G_{11} G_{21}+V_{11, \infty} G_{21}^{2}+V_{22, \infty}\right)$,

$$
b=\frac{1}{\left|V_{\infty}\right|}\left[V_{22, \infty} G_{11} G_{12}-V_{12, \infty}\left(G_{11} G_{22}+G_{12} G_{21}\right)\right.
$$$$
\left.+V_{11, \infty} G_{21} G_{22}-V_{12}\right] \text {, }
$$

$c=\frac{1}{2\left|V_{\infty}\right|}\left(V_{22, \infty} G_{12}^{2}-2 V_{12, \infty} G_{12} G_{22}+V_{11, \infty} G_{22}^{2}+V_{11, \infty}\right)$.

The marginal entropy for the thermal signal is

$R_{1}=R_{d, 1}+R_{s, 1}$

with

$R_{d, 1}=\frac{1}{2}\left[\ln \frac{V_{11, \infty}}{V_{11}}+\frac{V_{11}}{V_{11, \infty}}-1\right]$,

$R_{s, 1}=\frac{\varsigma_{1}^{2}}{2 V_{11, \infty}}$

and the average predictive power is

$\hat{P}_{1}=1-\exp \left(-R_{d, 1}\right) \cdot \frac{1}{2}\left(\left|V_{\infty}\right| a d\right)^{1 / 2}$,

with $d$ given by (57) and
$a=\frac{1}{2\left|V_{\infty}\right|}\left(V_{22, \infty} G_{11}^{2}+V_{22, \infty}\right)$,

$b=\frac{1}{\left|V_{\infty}\right|}\left(V_{22, \infty} G_{11} G_{12}-V_{12, \infty}\right)$,

$c=\frac{1}{2\left|V_{\infty}\right|}\left(V_{22, \infty} G_{12}^{2}+V_{11, \infty}\right)$.

Similar expressions can be derived for the univariate saline signal.

\section{References}

Barlas N, Josic K, Lapin S, Timofeyev I (2007) Non-uniform decay of predictability and return of skill in stochastic oscillatory models. Physica D 232:116-127

Barsugli JJ, Battisti DS (1998) The basic effects of atmosphere-ocean thermal coupling on midlatitude variability. J Atmos Sci 55: 477-493

Biastoch A, Böning CW, Getzlaff J, Molines JM, Madec G (2008) Causes of interannual-decadal variability in the meridional overturning circulation of the midlatitude North Atlantic Ocean. J Clim 21:6599-6615

Bjerknes J (1964) Atlantic air-sea interaction. Adv Geophys 10:1-82

Broecker WS, Bond G, Klas M (1990) A salt oscillator in the glacial Atlantic? 1. The concept. Paleoceanography 5(4):469-477

Bryan F (1987) Parameter sensitivity of primitive equation ocean general circulation models. J Phys Oceanogr 17:970-985

Chang P, Saravanan R, Delsole T, Wang F (2004) Predictability of linear coupled systems. Part I: theoretical analyses. J Clim 17:1474-1486

Cheng W, Bleck R, Rooth C (2004) Multi-decadal thermohaline variability in an ocean-atmosphere general circulation model. Clim Dyn 22:573-590

Clark PU, Pisias NG, Stocker TF, Weaver AJ (2002) The role of the thermohaline circulation in abrupt climate change. Nature 415:863-869

Collins M, Botzet M, Carril AF, Drange H, Jouzeau A, Latif M, Masina S, Otteraa OH, Pohlmann H, Sorteberg A, Sutton R, Terray L (2006) Interannual to decadal climate predictability in the North Atlantic: a multimodel-ensemble study. J Clim 19:1195-1203

Cover TM, Thomas J (1991) Elements of information theory. Wiley

Curry R, Mauritzen C (2005) Dilution of the northern North Atlantic Ocean in recent decades. Science 308:1772-1774

Dai A, Hu A, Meehl GA, Washington WM, Strand WG (2005) Atlantic thermohaline circulation in a coupled general circulation model: unforced variations versus forced changes. J Clim 18:3270-3293

Danabasoglu G (2008) On multidecadal variability of the Atlantic meridional overturning circulation in the community climate system model version 3. J Clim 21:5524-5544

DelSole T (2004) Predictability and information theory. Part I: measures of predictability. J Atmos Sci 61:2425-2440

DelSole T, Tippett MK (2007) Predictability: recent insights from information theory. Rev Geophys. doi:10.1029/2006RG000202

Delworth TL, Greatbatch RJ (2000) Multidecadal thermohaline circulation variability driven by atmospheric surface flux forcing. J Clim 13:1481-1495 
Delworth TL, Mann ME (2000) Observed and simulated multidecadal variability in the Northern Hemisphere. Clim Dyn 16:661-676

Delworth TL, Manabe S, Stouffer RJ (1993) Interdecadal variations of the thermohaline circulation in a coupled ocean-atmosphere model. J Clim 6:1993-2011

Delworth TL, Manabe S, Stouffer RJ (1997) Multidecadal climate variability in the Greenland Sea and surrounding regions: a coupled model simulation. Geophys Res Lett 24:257-260

Delworth TL, Zhang R, Mann ME (2007) Decadal to centennial variability of the Atlantic from observations and models. Geophys. Monog Am Geophys Union 173:131-148

Deser C, Blackmon ML (1993) Surface climate variations over the North Atlantic Ocean during winter: 1900-1989. J Clim 6:1743-1753

Dickson RR, Yashayaev I, Meincke J, Turrell WR, Dye S, Holfort J (2002) Rapid freshening of the deep North Atlantic Ocean over the past four decades. Nature 416:832-837

Dijkstra HA, Ghil M (2005) Low-frequency variability of the largescale ocean circulation: a dynamical systems approach. Rev Geophys 43. doi:10.1029/2002RG000122

Dima M, Lohmann G (2007) A hemispheric mechanism for the Atlantic multidecadal oscillation. J Clim 20:2076-2719

Dong B, Sutton RT (2005) Mechanism of interdecadal thermohaline circulation variability in a coupled ocean-atmosphere GCM. J Clim 18:1117-1135

Eden C, Greatbatch R (2002) Prospects for the decadal prediction of the North Atlantic Oscillation (NAO). Geophys Res Lett 29(10). doi:10.1029/2001GL014069

Enfield DB, Mestas-Nunez AM, Trimble PJ (2001) The Atlantic multidecadal oscillation and its relation to rainfall and river flows in the continental US. Geophys Res Lett 28:2077-2080

Folland CK, Parker DE, Kates FE (1984) Worldwide marine temperature fluctuations 1856-1981. Nature 310:670-673

Folland CK, Palmer TN, Parker DE (1986) Sahel rainfall and worldwide sea temperatures, 1901-85. Nature 320:602-607

Gardiner CW (1985) Handbook of stochastic methods, for physics, chemistry, and the natural sciences. Springer, Berlin, p 442

Gray ST, Graumlich LJ, Betancourt JL, Pederson GT (2004) A treering based reconstruction of the Atlantic Multidecadal Oscillation since 1567 A.D. Geophys Res Lett 31:L12205. doi: 10.1029/2004GL019932

Greatbatch RJ, Zhang S (1995) An interdecadal oscillation in an idealized ocean basin forced by constant heat flux. J Clim 8:81-91

Griffies SM, Bryan K (1997a) Predictability of the North Atlantic multidecadal climate variability. Science 275:181-184

Griffies SM, Bryan K (1997b) A predictability study of simulated North Atlantic multidecadal variability. Clim Dyn 13:459-487

Griffies SM, Tziperman E (1995) A linear thermohaline oscillator driven by stochastic atmospheric forcing. J Clim 8:2440-2453

Grotzner A, Latif M, Timmermann A (1999) Interannual to decadal predictability in a coupled ocean-atmosphere general circulation model. J Clim 12:2607-2624

Hakkinen S (1993) An Arctic source for the great salinity anomaly: a simulation of the Arctic ice-ocean system for 1955-1975. J Geophys Res 98:16397-16410

Hansen DV, Bezdek HF (1996) On the decadal anomalies in North Atlantic sea surface temperature. J Geophys Res 101:8749-8758

Hasselmann K (1976) Stochastic climate models: Part I. Theory. Tellus 28:473-485

Holland MM, Bitz CM, Eby M, Weaver AJ (2001) The role of iceocean interactions in the variability of the North Atlantic thermohaline circulation. J Clim 14:656-675

Huck T, Colin de Verdière A, Weaver AJ (1999) Interdecadal variability of the thermohaline circulation in box-ocean models forced by fixed surface fluxes. J Phys Oceanogr 29:865-892
Jungclaus JH, Haak H, Latif M, Mikolajewicz U (2005) Arctic-North Atlantic interactions and multidecadal variability of the meridional overturning circulation. J Clim 18:4013-4031

Kaplan A, Cane MA, Kushnir Y, Clement AC (1998) Analysis of global sea surface temperatures 1856-1991. J Geophys Res 103:18,575-18589

Kerr RA (2000) A North Atlantic climate pacemaker for the centuries. Science 288:1984-1986

Kleeman R (2002) Measuring dynamical prediction utility using relative entropy. J Atmos Sci 59:2057-2072

Kleeman R, Moore AM (1997) A theory for the limitation of ENSO predictability due to stochastic atmospheric transients. J Atmos Sci 54:753-767

Knight JR, Allan RJ, Folland CK, Vellinga M, Mann ME (2005) A signature of persistent natural thermohaline circulation cycles in observed climate. Geophys Res Lett 32:L20708. doi:10.1029/ 2005GL024233

Kushnir Y (1994) Interdecadal variations in North Atlantic sea surface temperature and associated atmospheric conditions. J Clim 7:141-157

Latif M, Roeckner E, Botzet M, Esch M, Haak H, Hagemann S, Jungclaus J, Legutke S, Marsland S, Mikolajewicz U, Mitchell J (2004) Reconstructing, monitoring, and predicting multidecadalscale changes in the North Atlantic thermohaline circulation with sea surface temperature. J Clim 17:1605-1614

Lohmann G, Schneider J (1999) Dynamics and predictability of Stommel's box model. A phase space perspective with implications for decadal climate variability. Tellus A 51(2):326-336

Lorenz EN (1969) The predictability of a flow which possesses many scales of motion. Tellus 21:289-307

Lozier MS (2010) Deconstructing the conveyer belt. Science 328(5985):1507-1511. doi:10.1126/science.1189250

Marotzke J, Stone PH (1995) Atmospheric transports, the thermohaline circulation, and flux adjustments in a simple coupled model. J Phys Oceanogr 25:1350-1364

Mikolajewicz U, Maier-Reimer E (1990) Internal secular variability in an ocean general circulation model. Clim Dyn 4:145-156

Mysak LA, Stocker TF, Huang F (1993) Century-scale variability in a randomly forced two-dimensional thermohaline ocean model. Clim Dyn 8:103-116

Ou HW (2006) Meridional thermal field of a coupled oceanatmosphere system: a conceptual model. Tellus 58A:404-415

Ou HW (2007) Hydrological cycle and ocean stratification in a coupled climate system: a theoretical study. Tellus 59A:683-694

Ozawa H, Ohmura A, Lorenz RD, Pujol T (2003) The second law of thermodynamics and the global climate system: a review of the maximum entropy production principle. Rev Geophys 41:4/ 10182003. doi:10.1029/2002RG000113

Peixoto JP, Oort AH (1992) Physics of climate. American Institute of Physics, New York

Polyakov IV, Alekseev GV, Bekryaev RV, Bhatt US, Colony R, Johnson MA, Karklin VP, Walsh D, Yulin AV (2003) Long-term ice variability in arctic marginal seas. J Clim 16:2078-2085

Rahmstorf S (2002) Ocean circulation and climate during the past 120,000 years. Nature 419:207-214

Saravanan R, McWilliams JC (1997) Stochasticity and spatial resonance in interdecadal climate fluctuations. J Clim 10:2299_ 2320

Saravanan R, Danabasoglu G, Doney SC, McWilliams JC (2000) Decadal variability and predictability in the midlatitude oceanatmosphere system. J Clim 13:1073-1097

Schneider T, Griffies S (1999) A conceptual framework for predictability studies. J Clim 12:3133-3155

Scott RB (2003) Predictability of SST in an idealized, one-dimensional, coupled atmosphere-ocean climate model with stochastic forcing and advection. J Clim 16:323-335 
Serreze MC, Barrett AP, Slater AG, Woodgate RA, Aagaard K, Lammers RB, Steele M, Moritz R, Meredith M, Lee CM (2006) The large-scale freshwater cycle of the Arctic. J Geophys Res 111(C11010). doi:10.1029/2005JC003424

Sevellec F, Huck T, Jelloul MB (2006) On the mechanism of centennial thermohaline oscillations. J Mar Res 64:355-392

Smith DM, Cusack S, Colman AW, Folland CK, Harris GR, Murphy JM (2007) Improved surface temperature prediction for the coming decade from a global climate model. Science 317:796-799

Stocker TF, Mysak LA (1992) Climatic fluctuations on the century time scale: a review of high-resolution proxy data and possible mechanisms. Clim Change 20:227-250

Stommel H (1961) Thermohaline convection with two stable regimes of flow. Tellus 13:224-230

Stommel H, Csanady G (1980) A relation between the T-S curve and global heat and atmospheric water transports. J Geophys Res 85:495-501

Talley LD, McCartney MS (1982) Distribution and circulation of Labrador sea water. J Phys Oceanogr 12:1189-1205

Te Raa LA, Dijkstra HA (2002) Instability of the thermohaline ocean circulation on interdecadal time scales. J Phys Oceanogr 32:138-160

Te Raa LA, Dijkstra HA (2003) Modes of internal thermohaline variability in a single-hemispheric ocean basin. J Mar Res 61:491-516

Timmermann A, Latif M, Voss R, Groetzner A (1998) Northern hemisphere interdecadal variability: a coupled air-sea mode. J Clim 11:1906-1931
Ting MF, Kushnir Y, Seager R, Li CH (2009) Forced and internal twentieth-century SST trends in the North Atlantic. J Clim 22(6): 1469-1481

Tippins D, Tomczak M (2003) Meridional turner angles and density compensation in the upper ocean. Ocean Dyn 53:332-342

Tziperman E, Ioannou PJ (2002) Transient growth and optimal excitation of thermohaline variability. J Phys Oceanogr 32(12):3427-3435

Tziperman E, Zanna L, Penland C (2008) Non-normal thermohaline circulation dynamics in a coupled ocean-atmosphere GCM. J Phys Oceanogr 38:588-604

Walin G (1985) The thermohaline circulation and the control of ice ages. Palaeogeo Palaeoclim Palaeoeco 50:323-332

Wallace JM, Gutzler DS (1981) Teleconnections in the geopotential height field during the northern hemisphere winter. Mon Weather Rev 109:784-812

Walsh JE, Chapman WL (1990) Arctic contribution to upper ocean variability in the North Atlantic. J Clim 3:1462-1473

Winton M, Sarachik ES (1993) Thermohaline oscillations induced by strong steady salinity forcing of ocean general circulation models. J Phys Oceanogr 23:1389-1410

Wunsch C, Ferrari R (1994) Vertical mixing, energy, and the general circulation of the oceans. Annu Rev Fluid Mech 36:281-314. doi:10.1146/annurev.fluid.36.050802.122121 